\title{
Stability of the Malvinas Current
}

F.J. Beron-Vera, ${ }^{1, a)}$ N. Bodnariuk, ${ }^{2,3,4}$ M. Saraceno, ${ }^{2,3,4}$ M.J. Olascoaga, ${ }^{5}$ and C. Simionato $2,3,4$

1)Department of Atmospheric Sciences, Rosenstiel School of Marine and Atmospheric Science, University of Miami, Miami, Florida 33149, USA.

2) Centro de Investigaciones del Mar y la Atmósfera (CIMA/CONICET-UBA), Ciudad Autónoma de Buenos Aires C1428EGA, Argentina.

3) Departamento de Ciencias de la Atmósfera y los Océanos,

Facultad de Ciencias Exactas y Naturales, Universidad de Buenos Aires, Ciudad Autónoma de Buenos Aires C1428EGA, Argentina.

4) Unidad Mixta Internacional-Instituto Franco-Argentino para el Estudio del Clima y sus Impactos (UMI-IFAECI/CNRS-CONICET-UBA), Ciudad Autónoma de Buenos Aires C1428EGA, Argentina.

${ }^{5)}$ Department of Ocean Sciences, Rosenstiel School of Marine and Atmospheric Science, University of Miami, Miami, Florida 33149, USA.

(Dated: December 16, 2019) 
Deterministic and probabilistic tools from nonlinear dynamics are used to assess enduring near-surface Lagrangian aspects of the Malvinas Current. The deterministic tools are applied on a multi-year record of velocities derived from satellite altimetry data, revealing a resilient cross-stream transport barrier. This is composed of shearless-parabolic Lagrangian coherent structures (LCS), which, extracted over sliding time windows along the multi-year altimetry-derived velocity record, lie in near coincidental position. The probabilistic tools are applied on a large collection of historical satellite-tracked drifter trajectories, revealing weakly communicating flow regions as basins of attraction for long-time asymptotic almost-invariant sets on either side of the altimetry-derived barrier. Shearless-parabolic LCS are detected for the first time from altimetry data, and their significance is supported on satellitederived ocean color data, which reveal shapes that quite closely resemble the peculiar V shapes, dubbed "chevrons," that have recently confirmed the presence of similar LCS in the atmosphere of Jupiter. Finally, using available in-situ velocity and hydrographic data, sufficient and necessary conditions for nonlinear symmetric stability are found to be satisfied, suggesting a duality between Lagrangian and Eulerian stability for the Malvinas Current.

PACS numbers: 02.50.Ga; 47.27.De; 92.10.Fj

Keywords: LCS, almost invariant set, shearless, parabolic, satellite altimetry, drifter, ocean color, chevron, Eulerian/Lagrangian stability

\footnotetext{
a) Electronic mail: fberon@miami.edu
} 
Analysis of independent Lagrangian data using independent finite-dimensional dynamical systems analysis techniques provide consistent results for the Lagrangian coherence of a western boundary current in the ocean, the Malvinas Current. These results along with those from the analysis of Eulerian data using an infinite-dimensional dynamical systems analysis technique suggest a Lagrangian-Eulerian duality for this current, a nonobvious result by view of the known generic tendency of two-dimensional unsteady laminar flow to sustain irregular fluid particle motion.

\section{INTRODUCTION}

The Malvinas Current originates as a result of a pronounced northward turn of the northern edge of the Antarctic Circumpolar Current past the Drake Passage. Carrying within a substantial portion of the upper limb of the Atlantic Meridional Overturning Circulation ${ }^{1}$, it represents a northward pathway for nutrient-rich subpolar water, making the western margin of the Argentine Basin a region of enhanced biological activity ${ }^{2}$ and significant fisheries ${ }^{3}$. The Malvinas Current flows northward up to about $38^{\circ} \mathrm{S}$, where it sharply turns eastward upon meeting the southward-flowing Brazil Current to form the Brazil-Malvinas Confluence $^{4}$, a region characterized by high mesoscale variability ${ }^{5}$.

The body of work dealing with the dynamics of the Malvinas Current is now quite important ${ }^{6-14}$. Of particular relevance for our purposes is the work by Davis et al. ${ }^{15}$, who using Lagrangian observations suggested that the Malvinas Current is composed of a single, predominantly barotropic jet extending down to 750-m depth or more for most of its northward path along the western boundary as is constrained by potential vorticity conservation $^{16,17}$. High-resolution hydrographic data and direct current observations more recently suggested the presence of multiple baroclinic jets in addition to the main barotropic one $^{18}$, confirming earlier inferences made from the analysis of the surface thermal structure ${ }^{19}$.

The analysis of the surface thermal structure more specifically revealed regions of large temperature contrast along cores of high meridional velocity ${ }^{18}$. This finding is consistent with the expectation that the Malvinas Current should behave as barrier for crossstream transport. This expectation is motivated by behavior of jetstreams in the lower 
stratosphere $\mathrm{e}^{20-24}$ and the weather layer of Jupiter ${ }^{25,26}$ as well as earlier speculation that western boundary currents such as the Gulf Stream should behave as transport barriers ${ }^{27}$ and more recent work that has characterized zonal ocean currents as cross-stream mixing inhibitors ${ }^{28}$, which has been partially verified by applying heuristic analyses involving satellite altimetry data, drifter trajectories, and ocean color imagery ${ }^{29-32}$. Our goal in this paper is to test the above expectation and further assess its persistence over time.

To achieve our goal we use two types of tools from nonlinear dynamics, both especially designed to investigate global aspects of Lagrangian motion. One set of tools is deterministic, and build on geometric, observer-independent (or objective) notions of strain and shear. They target so-called Lagrangian coherent structures $(L C S)^{33}$ as organizers of the Lagrangian circulation. This is done by means of a collection of global variational principles that constitute the geodesic theory of $\mathrm{LCS}^{34-42}$. The deterministic tools are more effective when the velocity field is known as this can be integrated to generate the required flow map that needs to be subsequently differentiated with respect to initial positions.

The other set of tools considered is probabilistic. These tools root in ergodic theory and, under appropriate time-homogeneity assumptions, can unveil from the Lagrangian circulation statistically weak communicating flow regions that form the basis for the construction of Lagrangian geographies ${ }^{43-47}$. The theoretical foundation for this is provided by a series of results from the study of autonomous dynamical systems using probability densities that have led to the notion of almost-invariant sets ${ }^{48-52}$. Central to this approach is the PerronFrobenius (or transfer) operator ${ }^{53}$ and the transition matrix, its discrete version that defines a Markov chain ${ }^{54-56}$ on boxes covering the flow domain. The probabilistic tools do not require flow map differentiation and can be applied directly on Lagrangian trajectories that do not start simultaneously under the above assumptions.

The deterministic tools are applied on a multi-year record of velocities derived from satellite altimetry data, revealing a persisting cross-stream transport barrier associated with the Malvinas Current in the near surface ocean. This barrier is composed of shearless-parabolic LCS, which, extracted over sliding time windows along the multi-year altimetry-derived velocity record, lie in near-coincidental position. Shearless-parabolic LCS generalize the concept of twistless invariant KAM (Kolmogorov-Arnold-Moser) tori from time-periodic ${ }^{57,58}$ or quasiperiodic ${ }^{21,59}$ flows to finite-time-aperiodic flows. The probabilistic tools are applied on a large collection of historical satellite-tracked trajectories of drifters drogued at $15 \mathrm{~m}$, re- 
vealing statistically weak communicating flow regions on either side of the altimetry-derived barrier.

Shearless-parabolic LCS are detected for the first time from altimetry data, and their significance is supported on satellite-derived ocean color data. Patterns revealed in such data are found to organize into $\mathrm{V}$ shapes nearly axially straddling the altimetry-derived LCS consistent with their shearless-parabolic nature ${ }^{39}$. Such V shapes have been to the best of our knowledge only reported to develop by clouds in the atmosphere of Jupiter at the boundaries of its characteristic zonal strips ${ }^{60}$. The significance of the enduring cross-stream transport barrier which characterizes the Malvinas Current is independently supported on drifter data. Furthermore, in-situ velocity and hydrographic data are used to suggest a duality between Lagrangian and Eulerian stability for the current.

The rest of the paper follows the standard organization into a methods section, a results section, and a summary and conclusions section. The various types of data considered are described as they are employed. Finally, the online Supplementary Material provides additional details on the deterministic and probabilistic tools as well as on the Eulerian stability result considered, thereby making the paper quite selfcontained.

\section{METHODS}

\section{A. Deterministic tools.}

The deterministic procedure involved in shearless-parabolic LCS extraction is succinctly as follows (cf. Section A in the online Supplementary Information for an expanded discussion). Given an incompressible, two-dimensional velocity field $v(x, t)$, the fluid trajectory equation

$$
\dot{x}=v(x, t)
$$

is integrated over the time interval $\left[t_{0}, t\right]$ to form the Cauchy-Green tensor

$$
C_{t_{0}}^{t}\left(x_{0}\right)=\mathrm{D} F_{t_{0}}^{t}\left(x_{0}\right)^{\top} \mathrm{D} F_{t_{0}}^{t}\left(x_{0}\right)
$$

where $F_{t_{0}}^{t}$ is the flow map that takes initial positions $x_{0}$ at $t_{0}$ to positions $x\left(t ; x_{0}, t_{0}\right)$ at $t$. The eigenvalues and eigenvectors of this observer-independent (i.e., objective) measure of 
deformation, satisfying

$$
0<\lambda_{1}\left(x_{0}\right) \equiv \lambda_{2}\left(x_{0}\right)^{-1} \leq 1, \quad \xi_{1}\left(x_{0}\right) \perp \xi_{2}\left(x_{0}\right),
$$

are then computed.

Time- $t_{0}$ positions of shearless-parabolic $L C S$ are subsequently sought as material lines formed by chains of alternating segments of Cauchy-Green tensorlines everywhere tangent to $\xi_{1}\left(x_{0}\right)$ and $\xi_{2}\left(x_{0}\right)$, namely, curves $r(s)$ satisfying

$$
r^{\prime}=\xi_{i}(r), \quad i=1,2
$$

The $\xi_{1}\left(x_{0}\right)$ - and $\xi_{2}\left(x_{0}\right)$-line segments are chosen:

1. to connect wedge and trisector singularities where $C_{t_{0}}^{t}\left(x_{0}\right)=\mathrm{Id}$, so the construction is structurally stable (i.e., robust under flow map perturbations); and

2. such that $\sqrt{\lambda_{1}\left(x_{0}\right)} \approx 1$ and $\sqrt{\lambda_{2}\left(x_{0}\right)} \approx 1$, respectively, so along-segment squeezing and stretching is kept close to neutral, thereby minimizing their hyperbolic nature.

A disk filled with tracer which is initially divided into two halves by one such shearlessparabolic LCS will, therefore, deform into a V shape axially straddling the LCS ${ }^{39}$. Observation of such behavior represents a more stringent test of the presence of a shearless-parabolic LCS with a cross-stream transport inhibitor effect than the observation of a large tracer contrast, which can be produced by LCS of any type.

\section{B. Probabilistic tools.}

Central to the probabilistic approach is a transition matrix $P \in \mathbb{R}^{N \times N}$ with components (cf. Section B in the online Supplementary Material for details)

$$
P_{i j}:=\operatorname{Pr}\left[\xi_{t+\mathcal{T}} \in B_{j} \mid \xi_{t} \in B_{i}\right]
$$

for a given transition time $\mathcal{T}$ and any time $t$, which provides a discrete representation of a transfer operator for the passive evolution on a domain $X$ of tracers governed by a timehomogeneous advection-diffusion process. Arguably, the time homogeneity assumption is appropriate for a probabilistic description of the dynamics, as done in statistically stationary turbulence (Orszag 1977). Yet it is also a consequence of the nature of the dataset 
on which the analysis is applied (the drifter data do not sample time sufficiently densely to enable the construction of a nonautonomous Markov chain). In any case its validity must be tested a posteriori, as we do, positively, here. (Similar positive tests of the validity of the time homogeneity assumption were produced by earlier work based on drifter data $^{43,44,46,47,61-63}$.) Drawn from a uniform distribution on $B_{i}, \xi_{t}$ is the initial position of a discrete-time trajectory $\left\{\xi_{t}, \xi_{t+\mathcal{T}}, \xi_{t+2 \mathcal{T}}, \ldots\right\}$ described by a tracer particle that randomly jumps, according to the stochastic kernel of the advection-diffusion process, between boxes in a collection $\left\{B_{1}, \ldots, B_{N}\right\}$ covering $X$. If $X$ is closed, then $\sum_{j=1}^{N} P_{i j}=1$ for every $i$, i.e., $P$ is row-stochastic. By construction, $P$ defines a Markov chain with states represented by the boxes of the partition. The discrete representation of a tracer probability density $f(x)$, i.e., satisfying $\int_{X} f(x) \mathrm{d} x=1$, is a probability vector $\mathbf{f}=\left(f_{1}, \cdots, f_{N}\right)$, where $f_{i}=\int_{B_{i}} f(x) \mathrm{d} x$ so $\sum_{i=1}^{N} f_{i}=1$. This evolves $k \mathcal{T}$ units of time according to

$$
\mathbf{f}^{(k)}=\mathbf{f} P^{k}, \quad k=1,2, \ldots .
$$

Long-time asymptotic aspects of the evolution on the Markov chain described $P$ can be inferred from its spectral properties ${ }^{49,51,64}$ as follows.

If $P$ is irreducible (i.e., all states in the Markov chain communicate) and aperiodic (i.e., no state is revisited cyclically), its dominant left eigenvector, $\mathbf{p}$, satisfies $\mathbf{p} P=\mathbf{p}$, can be chosen (componentwise) positive, and (scaled appropriately) represents a limiting invariant distribution, namely, $\mathbf{p}=\lim _{k \uparrow \infty} \mathbf{f} P^{k}$ for any probability vector $\mathbf{f}$ (cf., e.g., Horn and Johnson $^{55}$ ). In particular, $\mathbf{f}=\mathbf{1} / N$, where $\mathbf{1}=P \mathbf{1}$ is the right eigenvector corresponding to p.

The left eigenvector of $P$ with $\lambda$ closest to $1, \mathbf{l}_{2}$, is a signed vector which decays at the slowest rate ${ }^{65,66}$. Sets where the magnitude of the components of $\mathbf{l}_{2}$ maximize are the most dynamically disconnected as a random walker starting there will take the longest time to transit to other sets. The corresponding right eigenvector, $\mathbf{r}_{2}$, is also a signed vector, but it typically includes well-defined plateaus. A random trajectory conditioned on starting in a set forming the support of a plateau is expected to distribute in the long run, albeit only temporarily, as $\mathbf{l}_{2}$ where it takes a single like $\operatorname{sign}^{43,67}$. Such sets are therefore expected to form basins of attraction for time-asymptotic almost-invariant sets.

Decomposition of the ocean flow into weakly disjoint basins of attraction for almostinvariant attractors using the above spectral method has been shown to form the basis 
of a Lagrangian geography of the ocean, where the boundaries between basins are determined from the Lagrangian circulation itself, rather than from arbitrary geographical divisions ${ }^{43,44,46,47}$. The number of Lagrangian provinces will depend on the number of right eigenvectors considered. A large gap in the eigenspectrum of $P$ provides a cutoff criterion for eigenvector analysis, as provinces extracted from eigenvectors with eigenvalues on the right of the gap will have significantly shorter retention times than those extracted from eigenvectors on the left. (The eigenvector method we employ here differs from the flow network approach ${ }^{68,69}$ as the latter computes various graph-based quantities for finite-time durations to study flow dynamics, while the former analyzes time-asymptotic aspects of the dynamics through spectral information from the generating Markov chain.)

\section{RESULTS}

\section{A. Deterministic analysis.}

The deterministic tools are applied on a velocity field derived from sea-surface height $(\mathrm{SSH})$ in the region of interest, spanning $\left[70^{\circ} \mathrm{W}, 50^{\circ} \mathrm{W}\right] \times\left[55^{\circ} \mathrm{S}, 30^{\circ} \mathrm{S}\right]$. Specifically, we consider

$$
v(x, t)=g f^{-1} \nabla^{\perp} \eta(x, t)
$$

where $g$ is gravity, $f$ is the mean Coriolis parameter, and $\eta(x, t)$ is the superimposition on a mean dynamic topography ${ }^{70}$ of a daily $0.25^{\circ}$-resolution SSH anomaly field constructed from along-track satellite altimetry measurements ${ }^{71}$. Such an altimetry-derived velocity generally reflects an integral dynamic effect of the density field near the ocean surface, i.e., above the thermocline ${ }^{72}$. For the case of the Malvinas Current, it reflects quite well the velocity nearly over the entire water column ${ }^{11,73}$.

Figure 1 illustrates the application of the deterministic procedure on $t_{0}=12$ Dec 2001 with $T=t-t_{0}=-15$ days, which was kept fixed throughout. Here and in the calculations that we describe below, the integration of trajectories (1) and tensorlines (4) are carried out using a stepsize-adapting fourth-order Runge-Kutta method. Unlike trajectory integration, tensorline integration involves stepwise orienting the eigenvector field(s). Interpolations are obtained using a cubic scheme. Flow map differentiation in (2) is performed using finite differences. The Lagrangian coherence horizon $|T|=15$ days was selected such that 
sufficiently long shearless-parabolic LCS can be extracted. Long LCS impact transport more effectively than short LCS, which tend to coexist with a much longer LCS for this $|T|$ choice and thus are ignored. As $|T|$ is increased, singularities of the Cauchy-Green tensor tend to proliferate, resulting typically only in very short shearless-parabolic LCS with small effect on transport. The (time) integration direction, in turn, has been conveniently adopted to enable comparison with observed (e.g., satellite-derived) tracer distributions. More specifically, a tracer distribution at any given time is the result of the action of the flow on the tracer up to that time.

Depicted in the left panel of Fig. 1 is the longest shearless-parabolic LCS found in the domain. The nearly neutral squeezing and stretching Cauchy-Green tensorline segments that form the LCS are shown in blue and red, respectively. The wedge and trisector singularities connected by these segments are indicated by triangles and circles, respectively. The shearless-parabolic nature of the extracted LCS is demonstrated in the right panel of Fig. 1 , which shows, overlaid on the LCS (solid), the forward-advected image at time $t_{0}$ of three tracer disks axially straddling the backward-advected image of the LCS at $t_{0}-|T|$ (dashed). Note that the disks deform, as expected, into V shapes which very closely axially straddle the LCS at $t_{0}$.

Figure 2 shows a satellite-derived ocean pseudo-true color image on $t_{0}=12$ Dec 2001 with the extracted shearless-parabolic LCS overlaid. The coloration in the image is determined by the interactions of incident light with particles present in the water, mainly pigment chlorophyll, sediments, and dissolved organic material. Thus patterns formed in an ocean color image can be thought, to first approximation, as developed by a passive tracer. Note the various $\mathrm{V}$-shaped patches nearly axially straddling the extracted LCS. This provides strong independent observational support for the altimetry-inferred LCS and its shear-parabolic nature. Ocean color images showing $\mathrm{V}$ shapes of the type reported here are very rare; to the best of our knowledge we report their occurrence for the first time. (V-shaped structures should not be confused with mushroom-like structures, which are of hyperbolic nature ${ }^{74,75}$ and have long been known to exist in oceanography ${ }^{76-78}$.) Analogous $\mathrm{V}$ shapes, dubbed "chevrons," have been relatively recently observed in cloud distributions in the weather layer of Jupiter at the boundaries between so-called belts and zones organized around zonal jets $^{60}$. Jovian zonal jets have been rigorously characterized as shear-parabolic $\operatorname{LCS}^{26}$ and earlier heuristically as twistless KAM tori ${ }^{25}$. 
Shearless-parabolic LCS extraction was applied on sliding time windows $\left[t_{0}-|T|, t_{0}\right]$ with $t_{0}$ selected every two weeks since 15 Oct 2002 until 15 Sep 2005. During the period chosen, altimetry measurements were made by altimeters mounted on four satellites, thereby maximizing their availability and thus the quality of the derived velocity ${ }^{79,80}$.

The extracted shearless-parabolic LCS are depicted (in red) in Fig. 3 along with mean (over the LCS extraction period, 15 Oct 2002-15 Sep 2005) streamlines of the altimetryderived flow. The latter were selected to fill a strip around the Eulerian axis of the Malvinas Current, here taken as the streamline where the mean velocity maximizes at $42^{\circ} \mathrm{S}$. Note that LCS and streamlines do not coincide in position. Yet they run close inside the latitudinal band between about 38 and $49^{\circ} \mathrm{S}$. This suggests that the Malvinas Current behaves, within that latitudinal band, as a quasi-steady shearless-parabolic LCS. As such, it inhibits cross-stream transport persistently over time, largely preventing Patagonian shelf water from mixing with off-shelf water. The rather tightly packed collection of LCS forms the Lagrangian axis (or, more accurately, core) of the current.

The cross-stream barrier nature of the Malvinas Current is verified explicitly by the ensemble-mean evolution of tracers under the altimetry-derived flow. Selected snapshots are shown in Fig. 4. The ensemble-mean tracer evolution was computed by evolving the tracers from the same initial location on the shelf northeast of the Malvinas Islands, every two months over 15 Oct 2002-15 Sep 2005, and then computing on a daily basis during roughly half a year the percentage of tracer particles visiting each $0.75^{\circ}$-side box of a grid covering the domain. Note that the ensemble-mean tracer transport across the LCS is negligible.

The ensemble-mean tracer evolution in Fig. 4 reveals that practically all of the transport off the shelf takes place near $38^{\circ} \mathrm{S}$, where the collection of extracted shearless-parabolic LCS are interrupted, before most of them turn very briefly eastward and a few prolong a bit longer southeastward. The transport is directed eastward and then mainly southeastward, out of the domain through two exit routes, one at about $40^{\circ} \mathrm{S}$ and another one at $47^{\circ} \mathrm{S}$ or so. It is important to realize that this is not obvious from the inspection of the mean streamlines (Fig. 3), which suggest mainly eastward transport for a tracer released on the shelf at $38^{\circ} \mathrm{S}$, latitude at about which the Malvinas Current encounters the Brazil Current ${ }^{4}$. It must noted that recent observational and numerical work ${ }^{13,14,81}$ suggests that some fraction of the water carried by the Malvinas Current sinks at the Brazil-Malvinas Confluence and 
then flows northward underneath the Brazil Current. This process cannot be revealed by the deterministic analysis of altimetry data of this section (or the probabilistic analysis of drifter trajectories of the next section).

The close proximity of the shearless-parabolic LCS and the mean streamlines within 38 and $49^{\circ} \mathrm{S}$ (Fig. 3) suggests KAM-like behavior. In that latitudinal band, a decomposition of the flow into a steady (reference) component and a small unsteady (perturbation) component, certainly much smaller than near $38^{\circ} \mathrm{S}$ where mesoscale activity is known to be rather strong $^{5}$, may be envisioned as in earlier work ${ }^{82}$, in principle enabling a near-integrable Hamiltonian system stability analysis ${ }^{83}$. However, the flow is not recurrent, neither in time nor in space. In addition, quite unlike KAM curves, only portions of the reference Hamiltonian level sets (mean streamlines) are seen to "survive" under perturbation (i.e., when motion is produced by the total flow). These important differences indicate that ocean jets can sustain robust barriers for transport far beyond theoretical expectation ${ }^{59}$.

\section{B. Probabilistic analysis.}

The probabilistic tools are applied on daily interpolated trajectories produced by satellitetracked drifting buoys from the NOAA Global Drifter Program ${ }^{84}$ that have sampled the domain of interest. The drifter positions are satellite-tracked by the Argos system or GPS (Global Positioning System). The drifters follow the SVP (Surface Velocity Program) design, consisting of a surface spherical float which is drogued at $15 \mathrm{~m}$, to minimize wind slippage and wave-induced drift ${ }^{85}$. The drogue may not be present for the whole extent of a trajectory record ${ }^{86,87}$. We only consider trajectory portions during which the drogue is present, so a comparison with the altimetry-based results can be attempted.

We first cover the domain by $0.5^{\circ}$-side boxes. The size of the boxes was selected to maximize the grid's resolution while each individual box is sampled by enough drifters. Larger boxes would be sampled by more trajectories at the expense of making the Lagrangian provinces more leaky than they actually are due to the ensuing numerical diffusion ${ }^{88}$. Similar box sizes as used here have been considered earlier ${ }^{44-47,61}$ (robustness of the results presented below under box size variation is demonstrated in the top row of Fig. 4 in the online Supplementary Material). There are on average 28 drifters per box independent of the day over 1993-2013. Making the size of the boxes larger so more trajectories sample 
them leads to increased diffusion. The $(i, j)$ th component of the transition matrix $P$ in $(5)$ is estimated by counting the number of drifters which, visiting at any time $t$ box $B_{i}$, enter box $B_{j}$ at $t+\mathcal{T}$, and then dividing by the number of drifters in $B_{i}$.

We have set $\mathcal{T}=2$ days. This in general guarantees interbox communication. Furthermore, $\mathcal{T}=2$ days is longer than the Lagrangian decorrelation timescale, which has been estimated to be of about 1 day ${ }^{89}$. Markovian dynamics can be expected to approximately hold as there is negligible memory farther than 2 days into the past. A similar reasoning was applied in earlier applications involving drifter data ${ }^{44-47,61-63,90}$. Here the validity of the Markov model was estimated by checking that $\lambda(P(n \mathcal{T}))=\lambda(P(\mathcal{T}))^{n}$ holds well, particularly with $n$ up to at least 5 (cf. Fig. 3 in the online Supplementary Material). Consistent with this we have verified that the results presented below are largely insensitive to variations of $\mathcal{T}$ in the range 2-10 days (cf. bottom row of Fig. 4 in the online Supplementary Material).

We note that while the domain is open, $P$ has been constructed in such a way that it is row-stochastic by excluding all drifter trajectory pieces, which, starting inside the domain, terminate outside. It must be emphasized that this does not force trajectories to spuriously bounce back into the domain. The signature of inward motion is imprinted in the drifter trajectory data, so is in the resulting Markov-chain model. On the other hand, working with a row-stochastic $P$ facilitates the interpretation of the probabilistic tool results, albeit clearly not without exerting some care. Applying the Tarjan algorithm ${ }^{91}$ on the directed graph associated with the corresponding Markov chain reveals the existence of a set of boxes in the southwestern corner of the domain that are not reachable from boxes in its complement. The constructed $P$ is thus reducible. Nevertheless, the complement of that set of boxes covers most of the domain and furthermore is absorbing. So excluding it to make $P$ irreducible is inconsequential.

With the above in mind, we show in the top panel of Fig. 5 a portion of the eigenspectrum of $P$ corresponding to the largest 10 real eigenvalues. The largest eigenvalue equals unity and is simple. Consequently, the associated left eigenvector, which we loosely refer to as $\mathbf{p}$, is invariant, yet it is not strictly positive. The right eigenvector is $\mathbf{1}$. Any probability vector forward evolves under left multiplication by $P$ into $\mathbf{p}$, whose components maximize along the eastern boundary of the domain. More specifically, this happens inside the regions delimited by the black curves in the middle-left panel of Fig. 5. A tracer, irrespective of 
how it is initialized in the domain, will thus in the long run accumulate in those regions of the eastern boundary. Physically this means that it will eventually exit the domain through those locations.

The middle panel of Fig. 5 shows the left $\left(\mathbf{l}_{2}\right)$ and right $\left(\mathbf{r}_{2}\right)$ eigenvectors of $P$ corresponding to the second eigenvalue closest to $1\left(\lambda_{2}=0.9934\right)$. Note the two regions where the magnitude of the components $\mathbf{l}_{2}$ maximize. The support of these small regions represent almost-invariant attracting sets for tracers initially distributed on the large regions where $\mathbf{r}_{2}$ takes constant values, which represent their basins of attraction. (The eigenvectors have been arbitrary assigned signs such that regions of $\mathbf{r}_{2}$ evolve to like signed regions of $\mathbf{l}_{2}$.) These almost-invariant attracting sets, centered at about 38.5 and $48^{\circ} \mathrm{S}$ at the eastern side of the domain, physically represent routes of escape out of the domain for tracers in the corresponding basins of attraction. Traces initially inside each basin will passively evolve toward the respective attractor, which, being almost invariant, will retain the tracers temporarily until they are eventually drained out of the domain. Thus while $\mathbf{p}$ indicates that tracers will eventually exit the domain through the eastern boundary, $\mathbf{l}_{2}$ reveals preferred exit paths depending on how they are initialized.

The eigenspectrum of $P$ in the portion shown in the top panel of Fig. 5 reveals a gap between $\lambda_{2}$ and $\lambda_{3}$. Indeed, there is a drop of $2.1702 \%$ from $\lambda_{2}$ to $\lambda_{3}$, while $\lambda_{1}$ and $\lambda_{2}$ only differ by $0.0066 \%$ and $\lambda_{3}$ through $\lambda_{5}$ are very similar, changing by just $0.0091 \%$ on average. This suggests that a minimal significant Lagrangian geography with sufficiently large weakly communicating provinces to substantively constrain connectivity in the domain can be constructed by inspecting $\mathbf{r}_{2}$. A geography composed of smaller and less isolated provinces may be obtained by inspecting additional right eigenvectors, but this is not pursued here as our interest is to independently verify the deterministic analysis of the altimetry data, which suggested weak communication between shelf water on the west of the Malvinas Current and the open-ocean water on the east.

Shown in Fig. 6 is the constructed minimal Lagrangian geography. It includes three provinces, which are defined as follows. Rather than defining the Lagrangian provinces as sets where $\mathbf{r}_{2}$ takes one sign, as done in earlier work $^{43,44,46,47}$, here we define them as sets where the retention probability is maximized. More specifically, let $\mathcal{A} \subset\{1, \ldots, N\}$ and define $A:=\bigcup_{i \in \mathcal{A}} B_{i} \subset X$. If one conditions on a tracer trajectory to start in set $A$, the probability (relative to the invariant measure, $\mathbf{p}$ ) to be retained within $A$ after 
one application of $P$ is $\rho(A):=\sum_{i, j \in \mathcal{A}} p_{i} P_{i j} / \sum_{i \in \mathcal{A}} p_{i}{ }^{49,51}$. The bottom panel shows $\rho$ for $A(c):=\bigcup_{i: \mathbf{r}_{2}<c} B_{i}$ if $c<0$ or $\bigcup_{i: \mathbf{r}_{2}>c} B_{i}$ if $c>0$. We compute $\max _{c} \rho(A(c))=0.9835$ and 0.9763 at $c=c_{-}=-0.0016$ and $c=c_{+}=0.0219$, respectively (Table I). The sets $A_{\text {blue }}:=\bigcup_{i: \mathbf{r}_{2}<c_{-}} B_{i}$ and $A_{\text {red }}:=\bigcup_{i: \mathbf{r}_{2}>c_{+}} B_{i}$, depicted red and blue in Fig. 6, respectively, form the main Lagrangian provinces. The set depicted yellow, $A_{\text {yellow }}:=\bigcup_{i: c_{-}<\mathbf{r}_{2}<c_{+}} B_{i}$, represents a transition province with smaller retention probability, $\rho\left(A_{\text {yellow }}\right)=0.7797$. (We note that the transition province is revealed in the third subdominant right eigenvector, $\mathbf{r}_{4}$, and that the sparse eigenbasis approximation (SEBA) algorithm ${ }^{92}$, an adapted form of sparse principal component analysis by rotation and truncation ${ }^{93}$, reveals it from $\mathbf{r}_{2}$.)

Larger (smaller) retention probability is associated with longer (shorter) retention time. A simple measure of retention time is computed as follows. Consider $\left.\mathbf{p}_{A} P\right|_{A}=\lambda_{A} \mathbf{p}_{A}$, where $\left.P\right|_{A}$ is $P$ restricted to some set $A \subset X$ and $\lambda_{A}$ is the largest eigenvalue of $\left.P\right|_{A}$. If $P$ is irreducible, $\lambda_{A}<1$ and $\mathbf{p}_{A} \geq 0$. Assume that a tracer trajectory starts in $A$. If the trajectory is conditioned on being retained in $A$, it will asymptotically distribute as $\mathbf{p}_{A}$, where $\mathbf{p}_{A}$ has been normalized to a probability vector. Such a $\mathbf{p}_{A}$ is called a quasi-stationary distribution (cf. Chapter 6.1.2 of Bremaud ${ }^{94}$ ). The expectation of the random time to exit $A$ is $\tau(A):=\mathcal{T} /\left(1-\lambda_{A}\right)$ (cf. Section B.7 in the online Supplementary Material). Such a $\tau(A)$ provides an average measure of retention time in $A$. We compute $\tau\left(A_{\text {blue }}\right) \approx 11$ months and $\tau\left(A_{\text {red }}\right) \approx 8$ months for the main Lagrangian provinces, and a much shorter retention time, $\tau\left(A_{\text {yellow }}\right) \approx 1$ month, for the transition province (Table $\mathrm{I}$ ).

Clearly, the partition of the flow domain provided by the drifter-based Lagrangian geography is indicative of low connectivity between shelf water and open-ocean water off the shelf, south of $38^{\circ} \mathrm{S}$. Figure 7 provides confirmation for this inference from direct calculation. More specifically, this figure shows selected snapshots of the evolution under left multiplication by $P$ of a tracer probability initially on the shelf, northeast of the Malvinas archipelago. Note that up to day 56 , the tracer probability propagates northeastward, predominantly confined within the transition province of the Lagrangian geography. The almost-invariant character of the boundaries of the Lagrangian provinces explains the small leakage of probability over the main provinces east and west of the transition province.

The leakage continues past day 56 of evolution, becoming stronger as the Brazil-Malvinas Confluence near $38^{\circ} \mathrm{s}$ is reached. Time-asymptotically the probability that leaks to the west and east of the transition province accumulates in the southern and northern almost- 
invariant attractor, respectively (cf. Fig. 5, middle-left panel). These attractors, we reiterate, physically represent exit routes out of the domain.

We note that if the tracer probability were initialized inside the transition province of the Lagrangian geography where this turns (south)eastward at about $38^{\circ} \mathrm{s}$, it would remain confined within for a shorter period of time before leaking out and being absorbed into the nearest almost-attracting set. The reason for this is the much closer proximity of the attractors to the transition province at these latitudes. The retention time measure $\tau\left(A_{\text {yellow }}\right)=1.1518$ months, discussed above, is an average measure for the entire transition province. The average retention time within the portion of transition province that lies (roughly) inside the Brazil-Malvinas Confluence region is somewhat smaller than 1 week. By contrast, the average retention time in the complement of this set is nearly 5 weeks, which is very close to the average retention time in the whole transition province. This is consistent with the behavior just described. Also consistent with this is the strong mesoscale variability that affects the area where the Malvinas and Brazil currents meet. Diffusion is benefited from such variability, which contributes to shorten the retention time there.

To assess the latter, one can leverage on the computation of the flux across the boundary of a set $A\left(=\bigcup_{i \in \mathcal{A}} B_{i}\right)$, which is readily accomplished as follows ${ }^{95}$. Let $\partial \mathcal{A}(\subset \mathcal{A})$ be the index set of boxes on the boundary of $A$. The flux $\Phi\left(B_{j}\right)$ through a boundary box $B_{j}$, with $j \in \partial \mathcal{A}$, can be calculated as $\Phi\left(B_{j}\right)=\Phi_{\text {out }}\left(B_{j}\right)-\Phi_{\text {in }}\left(B_{j}\right)$, where $\Phi_{\text {out }}\left(B_{j}\right)=\mathcal{T}^{-1}$. $\operatorname{vol}\left(B_{j}\right) \sum_{k \in\{1, \ldots, N\} \backslash \mathcal{A}} P_{j k}$ and $\Phi_{\text {in }}\left(B_{j}\right)=\mathcal{T}^{-1} \cdot \sum_{k \in\{1, \ldots, N\} \backslash \mathcal{A}} \operatorname{vol}\left(B_{k}\right) P_{k j}$. Figure 8 shows an evaluation of the flux formulas for the transition province $\left(A_{\text {yellow }}\right)$, with $\operatorname{vol}\left(B_{i}\right)$ estimated as area $\left(B_{i}\right) \cdot H$ where $H=15 \mathrm{~m}$ is the drogue depth. Note that the flux through the boundary boxes of these sets tend to maximize inward or outward in the Brazil-Malvinas Confluence region.

We note finally that despite the limitation provided by the number of drifters available, particularly over the continental shelf, the inferred Lagrangian provinces are in very good agreement with the biophysical provinces deduced by Longhurst ${ }^{2}$ and more recently by Saraceno et al. ${ }^{96,97}$ using two independent methods. 


\section{Synthesis of deterministic and probabilistic analyses.}

The results of the deterministic analysis of the altimetry data and the probabilistic analysis of the drifter data are largely consistent. They both independently indicate Lagrangian stability for the Malvinas Current, which largely behaves as a barrier that prevents shelf water to its west from being mixed with off-shelf water to its east. This is well demonstrated in Fig. 6, which shows that the shearless-parabolic LCS extracted from altimetry over sliding time windows along the multiyear record analyzed lie well within the transition province between the main Lagrangian provinces constructed from all available drifter data.

The large majority of the water transported by the Malvinas Current leaves the shelfbreak near $38^{\circ} \mathrm{S}$, where the Malvinas Current meets the Brazil Current (cf. Figs. 4 and 7). The Brazil-Malvinas Confluence region is characterized by strong mesoscale variability, which the probabilistic analysis showed to promote diffusion in the region. Consistent with this, the deterministic analysis revealed LCS prolonging only briefly southeastward at the BrazilMalvinas Confluence latitude, thereby allowing unrestrained exchanges there. According to both the deterministic and probabilistic results, the off-shelf export eventually reaches the South Atlantic's interior (east of $50^{\circ} \mathrm{W}$ ) through two routes, centered at about 40 and $47^{\circ} \mathrm{S}$.

\section{Lagrangian-Eulerian stability duality.}

The reported Lagrangian stability of the Malvinas Current motivates the question of its stability in the Eulerian frame. A stability result for a general meandering meridional current with vertical shear is lacking. Yet the stability of a basic flow (steady solution) $V(x, z)$ in thermal-wind balance, where $x$ is cross-stream and $-z$ depth, of the $y$-independent, inviscid, unforced, nonhydrostatic, Boussinesq equations on an $f$ plane is well established ${ }^{98,99}$. Both sufficient and necessary conditions for the symmetric stability of $V(x, z)$ under arbitrarily large and shaped perturbations are given by $N^{2} /\left(\partial_{z} V\right)^{2}>1 /\left(1+\partial_{x} V / f\right)>0$, where $N^{2}$ is the square of the basic flow's Brunt-Väisälä frequency. Note that symmetric stability requires both static stability $\left(N^{2}>0\right)$ as well as inertial stability $\left.\left(f^{2}+f \partial_{x} V\right)>0\right)$. Assuming stable stratification, these conditions are equivalent to $f Q>0$, where $Q:=N^{2}\left(f+\partial_{x} V\right)-f\left(\partial_{z} V\right)^{2}$ is the basic flow's potential vorticity, which is materially preserved. Clearly, $f Q<0$ is both necessary and sufficient for symmetric instability. This condition includes the necessary 
condition for instability under infinitesimally small normal-mode perturbations originally derived by Hoskins ${ }^{100}$ (cf. Section C in the online Supplementary Material for a review of the results just described).

Using available direct high-resolution velocity measurements and temperature and salinity data collected by RSS Discovery in late December 1992 during WOCE cruise A11 along $45^{\circ} \mathrm{S}^{101}$, we proceed to check if the Malvinas Current has any hope to be symmetrically stable. The WOCE-A11 transect lies across the Malvinas Current, which we assume to be represented as an along-stream-symmetric sheared flow. The velocity data was collected by a hull mounted acoustic Doppler current profiler (ADCP) in a westward course. A section of meridional (nearly along-stream) velocity is shown in the top-left panel of Fig. 9. The temperature and salinity were obtained from conductivity-temperature-depth (CTD) casts occupied in a returned eastward course; the Brunt-Väisälä frequency, averaged along the section, is shown in the top-right panel Fig. 9. The bottom-left and bottom-right panels of Fig. 9 show along-section-mean $1 /\left(1+\partial_{x} V / f\right)$ and $N^{2} /\left(\partial_{z} V\right)^{2}$, respectively. Note that the symmetric stability conditions are well satisfied on average across the Malvinas Current. This result together with those from the deterministic and probabilistic nonlinear dynamics analysis suggest a Lagrangian-Eulerian stability duality for the current.

The above result is not obvious whatsoever. Indeed, it is well-known that unsteady laminar Eulerian flows can support irregular Lagrangian motion (chaotic Lagrangian motion generically in bounded, recurrent unsteady two-dimensional flows ${ }^{102}$. But there are several caveats to have in mind. First, a priori conditions for stability/instability should be verified by the basic flow rather than the total flow and instantaneously as we have checked here. Yet Piola et al. ${ }^{18}$ note that the ADCP velocity shear in the 100- through 390-m depth interval is virtually identical to the geostrophic shear derived from hydrography. This suggests that the ADCP velocity may be providing a reasonable representation of the basic velocity. Also, hydrographic data are not available with enough longitudinal resolution to check the symmetric stability conditions pointwise. And last but not least, there are not sufficient data to assess the extent to which along-stream symmetry holds for the Malvinas Current. This has most chances to be verified within $38-49^{\circ} \mathrm{S}$, (co)incidentally where shearless-parabolic LCS and the mean streamlines were found to lie closest together. 


\section{SUMMARY AND FINAL REMARKS}

In this paper we have characterized the Malvinas Current as an enduring cross-stream transport barrier by applying nonlinear dynamics tools of two quite different types on independent datasets.

One type of tools used was deterministic, built on a geometric, objective (i.e., observerindependent) notion of material shear. These tools were applied on velocities derived from satellite altimetry, and revealed - for the first time from this dataset-Lagrangian coherent structures (LCS) of the shearless-parabolic class. Computed over sliding time windows along a multiyear period of satellite altimetry data with the highest density, the shearless-parabolic LCS were found to form an enduring near-surface Lagrangian axis for the Malvinas Current that largely inhibits shelf water on its western side from mixing with open-ocean water on its eastern side.

The other type of nonlinear dynamics tools employed was probabilistic, built on ergodic theory and describing tracer motion on a Markov chain. These were applied on available satellite-tracked drifter trajectories, revealing statistically weak communicating Lagrangian provinces separated by the LCS extracted from altimetry. This provided independent support for the enduring role of the Malvinas Current in the near surface as a cross-stream transport barrier.

The shear-parabolic nature of the Lagrangian axis of the Malvinas Current was supported on satellite-derived ocean color imagery. This revealed - for the first time to the best of our knowledge - V shapes nearly axially straddling current's Lagrangian axis. Similar V shapes, referred to as "chevrons," have been been relatively recently observed in clouds distributions in the weather layer of Jupiter, confirming the enduring nature of zonal jets there as barriers for meridional transport.

In-situ velocity and hydrographic data showed that conditions for symmetric stability are satisfied. This suggested a Lagrangian-Eulerian stability duality for the Malvinas Current, a nonobvious result given the known ability of laminar Eulerian flows to support irregular Lagrangian motion.

It is important to stress that these results do not imply that the Malvinas Current is a perfect transport barrier. Indeed, while they do suggest overall stability in a LagrangianEulerian sense for the Malvinas Current, they do not rule out the possibility of instantaneous 
cross-stream intrusions/extrusions, which are supported by observations ${ }^{17}$. A number of physical mechanisms may be responsible for them including wind stress curl and induced upwelling ${ }^{96,103,104}$, and internal waves ${ }^{3,7,96,105}$ and other submesoscale processes ${ }^{106}$.

Finally, the gas giant's chevrons have been connected to inertia-gravity wave motion ${ }^{60}$. Satellite imagery has recently revealed internal waves propagating along the Patagonian shelfbreak and continental slope in the opposite direction of the Malvinas Current ${ }^{107}$. The possible connection with the chevrons observed along the Lagrangian axis of the current deserves to be investigated. This is beyond the scope of this paper as also is investigating how representative the results here presented are of other western boundary currents. For instance, high-resolution measurements across the Gulf Stream suggest that symmetric stability is violated locally along submesoscale fronts in the upper ocean ${ }^{108}$, which already indicates a potentially important difference with the Malvinas Current.

\section{SUPPLEMENTARY MATERIAL}

The Supplementary Material includes three appendices providing additional details on the deterministic (A) and probabilistic (B) tools employed in the paper as well as on the Eulerian stability result considered $(\mathrm{C})$. This is done with a goal in mind of making the paper sufficiently selfcontained.

\section{ACKNOWLEDGMENTS}

We thank Peter Koltai for the benefit of discussions on transfer operators defined using stochastic kernels and Markov chains, and Daniel Karrasch for the benefit of discussions on line fields. We also thank Joaquin Triñanes for helping us finding the MODIS-Terra ocean psuedocolor image in Fig. 2. MODIS-Terra data are available from NASA's OceanColor Web (https://oceancolor.gsfc.nasa.gov) with support from the Ocean Biology Processing Group (OBPG) at NASA's Goddard Space Flight Center. The altimeter products were produced by SSALTO/DUCAS and distributed by AVISO with support from CNES (http:// www.aviso.oceanobs). The drifter data are available from the NOAA Global Drifter Program (http://www.aoml.noaa.gov/phod/dac). This work has been supported by ONR Global grant 12275382 . 


\section{REFERENCES}

${ }^{1}$ Y. Friocourt, S. Drijfhout, B. Blanke, and S. Speich, "Water mass export from Drake Passage to the Atlantic, Indian, and Pacific Oceans: A Lagrangian model analysis," J. Phys. Oceanogr. 35, 1206-1222 (2005).

${ }^{2}$ A. Longhurst, Ecological Geography of the Sea (Academic Press, San Diego., San Diego, 1998) p. 560.

${ }^{3}$ E. M. Acha, H. W. Mianzan, R. A. Guerrero, M. Favero, and J. Bava, "Marine fronts at the continental shelves of austral South America: Physical and ecological processes," J. Mar. Syst. 44, 83-105 (2004).

${ }^{4}$ R. P. Matano, "On the separation of the Brazil Current from the coast," Journal of Physical Oceanography 23, 79-90 (1993).

${ }^{5}$ G. J. Goni, S. Kamholz, S. L. Garzoli, and D. B. Olson, "Dynamics of the Brazil/Malvinas Confluence based on inverted echo sounders and altimetry," J. Geophys. Res. 101, 1627316289 (1996).

${ }^{6}$ D. B. Olson, G. P. Podesta, R. H. Evans, and O. B. Brown, "Temporal variations in the separation of Brazil and Malvinas Currents," Deep Sea Research Part A. Oceanographic Research Papers 35, 1971-990 (1988).

${ }^{7}$ F. Vivier, C. Provost, and M. P. Meredith, "Remote and local forcing in the BrazilMalvinas region,” J. Phys. Oceanogr. 31, 892-913 (2001).

${ }^{8}$ R. P. Matano and E. D. Palma, "On the upwelling of downwelling currents," J. Phys. Oceanogr. 38, 2482-2500 (2008).

${ }^{9}$ V. Combes and R. P. Matano, "The Patagonian shelf circulation: Drivers and variability," Progress in Oceanography 167, 24-43 (2018).

${ }^{10}$ B. C. Franco, E. D. Palma, V. Combes, and M. L. Lasta, "Physical processes controlling passive larval transport at the patagonian shelf break front," Journal of Sea Research 124, 17-25 (2017).

${ }^{11}$ R. Ferrari, C. Artana, M. Saraceno, A. R. Piola, and C. Provost, "Satellite altimetry and current-meter velocities in the Malvinas Current at $41^{\circ} \mathrm{s}$ : Comparisons and modes of variations," Journal of Geophysical Research: Oceans 122, 9572-9590 (2017), https://agupubs.onlinelibrary.wiley.com/doi/pdf/10.1002/2017JC013340. 
${ }^{12}$ C. Artana, J.-M. Lellouche, Y. H. Park, G. Garric, Z. Koenig, N. Sennechael, R. Ferrari, A. R. Piola, M. Saraceno, and C. Provost, "Fronts of the Malvinas Current System: Surface and subsurface expressions revealed by aatellite altimetry, Argo Floats, and Mercator Operational Model outputs," Journal of Geophysical Research: Oceans 123, 5261-5285 (2018).

${ }^{13}$ C. Artana, R. Ferrari, Z. Koenig, N. Sennechael, M. Saraceno, A. R. Piola, and C. Provost, "Malvinas Current volume transport at $41^{\circ} \mathrm{s}$ : A 24 year long time series consistent with mooring data from 3 decades and satellite altimetry," Journal of Geophysical Research: Oceans 123, 378-398 (2018).

${ }^{14}$ G. F. Paniagua, M. Saraceno, A. R. Piola, R. Guerrero, C. Provost, R. Ferrari, L. S. Lago, and C. I. Artana, "Malvinas Current at $40^{\circ} \mathrm{s}-41^{\circ} \mathrm{s}$ : First assessment of temperature and salinity temporal variability," Journal of Geophysical Research: Oceans 123, 5323-5340 (2018).

${ }^{15}$ R. E. Davis, P. D. Killworth, and J. R. Blundell, "Comparison of Autonomous Lagrangian Circulation Explorer and fine resolution Antarctic model results in the South Atlantic," J. Geophys. Res. 101, 855-884 (1996).

${ }^{16}$ M. Saraceno, C. Provost, A. R. Piola, J. Bava, and A. Gagliardini, "Brazil Malvinas Frontal System as seen from 9 years of advanced very high resolution radiometer data," J. Geophys. Res. 109, C05027 (2004).

${ }^{17}$ A. R. Piola, N. M. Avellaneda, R. A. Guerrero, F. P. Jardón, E. D. Palma, and S. I. Romero, "Malvinas-slope water intrusions on the northern Patagonia continental shelf," Ocean Sci. 6, 345-359 (2010).

${ }^{18}$ A. R. Piola, B. C. Franco, E. D. Palma, and M. Saraceno, "Multiple jets in the Malvinas Current," J. Geophys. Res. 118, doi:10.1002/jgrc.20170. (2013).

${ }^{19}$ B. C. Franco, A. R. Piola, A. L. Rivas, A. Baldoni, and J. P. Pisoni, "Multiple thermal fronts near the Patagonian shelf break," Geophys. Res. Lett. 35, L02607 (2008).

${ }^{20}$ I. I. Rypina, M. G. Brown, F. J. Beron-Vera, H. Kocak, M. J. Olascoaga, and I. A. Udovydchenkov, "On the Lagrangian dynamics of atmospheric zonal jets and the permeability of the stratospheric polar vortex," J. Atmos. Sci. 64, 3595-3610 (2007).

${ }^{21}$ F. J. Beron-Vera, M. J. Olascoaga, M. G. Brown, H. Koçak, and I. I. Rypina, "Invarianttori-like Lagrangian coherent structures with application to geophysical flows," Chaos $\mathbf{2 0 ,}$ $017514(2010)$. 
${ }^{22}$ F. J. Beron-Vera, "The role of jets as transport barriers in the Earth's stratosphere," J. Phys.: Conf. Ser. 318, 072002 (2011).

${ }^{23}$ F. J. Beron-Vera, M. J. Olascoaga, M. G. Brown, and H. Koçak, "Zonal jets as meridional transport barriers in the subtropical and polar lower stratosphere," J. Atmos Sci. 69, 753767 (2012).

${ }^{24}$ M. J. Olascoaga, F. J. Beron-Vera, G. Haller, J. Trinanes, M. Iskandarani, E. F. Coelho, B. Haus, G. J. H. S. Huntley, A. D.Kirwan, Jr., B. L. Lipphardt, Jr., T. Özgökmen, A. J. H. M. Reniers, and A. Valle-Levinson, "Drifter motion in the Gulf of Mexico constrained by altimetric Lagrangian Coherent Structures," Geophys. Res. Lett. 40, 61716175 (2013).

${ }^{25}$ F. J. Beron-Vera, M. G. Brown, M. J. Olascoaga, I. I. Rypina, H. Koçak, and I. A. Udovydchenkov, "Zonal jets as transport barriers in planetary atmospheres," J. Atmos. Sci. 65, 3316-3326 (2008).

${ }^{26}$ A. Hadjighasem and G. Haller, "Geodesic transport barriers in Jupiter's atmosphere: a video-based analysis," SIAM Review 58, 69-89 (2016).

${ }^{27}$ A. S. Bower, H. T. Rossby, and J. L. Lillibridge, "The Gulf Stream-barrier or blender?" J. Phys. Oceanogr. 15, 24-32 (1985).

${ }^{28}$ R. Ferrari and M. Nikurashin, "Suppression of eddy diffusivity across jets in the Southern Ocean," Journal of Physical Oceanography 40, 1501-1519 (2010).

${ }^{29}$ J. Marshall, E. Shuckburgh, H. Jones, and C. Hill, "Estimates and implications of surface eddy diffusivity in the Southern Ocean derived from tracer transport," J. Phys. Oceanogr. 36, 1806-1821 (2006).

${ }^{30}$ F. J. Beron-Vera, "Mixing by low- and high-resolution surface geostrophic currents," J. Geophys. Res. 115, C10027 (2010).

${ }^{31}$ I. I. Rypina, L. J.Pratt, and M. S. Lozier, "Near-surface transport pathways in the North Atlantic Ocean: Looking for throughput from the Subtropical to the Subpolar Gyre," J. Phys. Oceanogr. 41, 911-925 (2011).

${ }^{32}$ F. Huhn, A. von Kameke, V. Pérez-Muñuzuri, M. J. Olascoaga, and F. J. Beron-Vera, "The impact of advective transport by the South Indian Ocean Countercurrent on the Madagascar plankton bloom," Geophys. Res. Lett. 39, L0662 (2012).

${ }^{33} \mathrm{G}$. Haller and G. Yuan, "Lagrangian coherent structures and mixing in two-dimensional turbulence," Physica D 147, 352-370 (2000). 
${ }^{34}$ G. Haller and F. J. Beron-Vera, "Geodesic theory of transport barriers in two-dimensional flows," Physica D 241, 1680-1702 (2012).

${ }^{35}$ M. Farazmand and G. Haller, "Attracting and repelling Lagrangian coherent structures from a single computation," Chaos 23, 023101 (2013).

${ }^{36}$ F. J. Beron-Vera, Y. Wang, M. J. Olascoaga, G. J. Goni, and G. Haller, "Objective detection of oceanic eddies and the Agulhas leakage," J. Phys. Oceanogr. 43, 1426-1438 (2013).

${ }^{37}$ G. Haller and F. J. Beron-Vera, "Coherent Lagrangian vortices: The black holes of turbulence," J. Fluid Mech. 731, R4 (2013).

${ }^{38}$ G. Haller and F. J. Beron-Vera, "Addendum to 'Coherent Lagrangian vortices: The black holes of turbulence'," J. Fluid Mech. 755, R3 (2014).

${ }^{39}$ M. Farazmand, D. Blazevski, and G. Haller, "Shearless transport barriers in unsteady two-dimensional flows and maps," Physica D 278-279, 44-57 (2014).

${ }^{40}$ D. Karrasch, F. Huhn, and G. Haller, "Automated detection of coherent Lagrangian vortices in two-dimensional unsteady flows," Proc. Royal Soc. A 471, 20140639 (2014).

${ }^{41}$ D. Karrasch, "Attracting Lagrangian coherent structures on Riemannian manifolds," Chaos 25, 087411 (2015).

${ }^{42}$ G. Haller, "Climate, black holes and vorticity: How on Earth are they related?" SIAM News 49, 1-2 (2016).

${ }^{43}$ G. Froyland, R. M. Stuart, and E. van Sebille, "How well-connected is the surface of the global ocean?" Chaos 24, 033126 (2014).

${ }^{44}$ P. Miron, F. J. Beron-Vera, M. J. Olascoaga, J. Sheinbaum, P. Pérez-Brunius, and G. Froyland, "Lagrangian dynamical geography of the Gulf of Mexico," Scientific Reports 7, 7021 (2017).

${ }^{45}$ M. J. Olascoaga, P. Miron, C. Paris, P. Pérez-Brunius, R. Pérez-Portela, R. H. Smith, and A. Vaz, "Connectivity of Pulley Ridge with remote locations as inferred from satellitetracked drifter trajectories," Journal of Geophysical Research 123, 5742-5750 (2018).

${ }^{46}$ P. Miron, F. J. Beron-Vera, M. J. Olascoaga, G. Froyland, P. Pérez-Brunius, and J. Sheinbaum, "Lagrangian geography of the deep Gulf of Mexico," J. Phys. Oceanogr. 49, 269290 (2019).

${ }^{47}$ P. Miron, F. J. Beron-Vera, M. J. Olascoaga, and P. Koltai, "Markov-chain-inspired search for MH370," Chaos: An Interdisciplinary Journal of Nonlinear Science 29, 041105 
(2019).

${ }^{48}$ M. Dellnitz and O. Junge, "Almost invariant sets in Chua's circuit," Internat. J. Bifur. Chaos 7, 2475-2485 (1997).

${ }^{49} \mathrm{M}$. Dellnitz and O. Junge, "On the approximation of complicated dynamical behavior," SIAM J. Numer. Anal. 36, 491-515 (1999).

${ }^{50} \mathrm{G}$. Froyland and M. Dellnitz, "Detecting and locating near-optimal almost-invariant sets and cycles," SIAM J. Sci. Comput. 24, 1839-1863 (2003).

${ }^{51}$ G. Froyland, "Statistically optimal almost-invariant sets," Physica D 200, 205-219 (2005).

${ }^{52}$ G. Froyland and K. Padberg, "Almost-invariant sets and invariant manifolds - connecting probabilistic and geometric descriptions of coherent structures in flows," Physica D 238, 1507-1523 (2009).

${ }^{53}$ A. Lasota and M. C. Mackey, Chaos, Fractals, and Noise: Stochastic Aspects of Dynamics, 2nd ed., Applied Mathematical Sciences, Vol. 97 (Springer, New York, 1994).

${ }^{54}$ J. G. Kemeny and J. L. Snell, Finite Markov Chains (Springer-Verlag, 1976).

${ }^{55}$ R. A. Horn and C. R. Johnson, Matrix Analysis (Cambridge University Press, 1990).

${ }^{56}$ J. Norris, Markov Chains (Cambridge University Press, 1998).

${ }^{57}$ D. del-Castillo-Negrete and P. J. Morrison, "Chaotic transport by Rossby waves in shear flow," Phys. Fluids A 5, 948-965 (1993).

${ }^{58}$ A. Delshams and R. de la Llave, "KAM theory and a partial justification of Greene's criterion for non-twist maps," SIAM J. Math. Anal. 31, 1,235-1,269 (2000).

${ }^{59}$ I. I. Rypina, M. G. Brown, F. J. Beron-Vera, H. Koçak, M. J. Olascoaga, and I. A. Udovydchenkov, "Robust transport barriers resulting from strong Kolmogorov-ArnoldMoser stability," Phys. Rev. Lett. 98, 104102 (2007).

${ }^{60}$ A. A. Simon-Miller, J. H. Rogers, P.J.Gierasch, D. Choi, M. D. Allison, G. Adamoli, and H.-J. Mettig, "Longitudinal variation and waves in Jupiter's south equatorial wind jet," Icarus 218, 817-830 (2012).

${ }^{61}$ F. J. Beron-Vera, M. J. Olascoaga, and P. Miron, "Building a Maxey-Riley framework for surface ocean inertial particle dynamics," Phys. Fluids 31, 096602 (2019).

${ }^{62}$ E. van Sebille, E. H. England, and G. Froyland, "Origin, dynamics and evolution of ocean garbage patches from observed surface drifters," Environ. Res. Lett. 7, 044040 (2012).

${ }^{63}$ R. McAdam and E. van Sebille, "Surface connectivity and interocean exchanges from drifter-based transition matrices," Journal of Geophysical Research: Oceans 123, 514- 
532 (2018).

${ }^{64}$ C. S. Hsu, Cell-to-cell mapping. A Method of Global Analysis for Nonlinear Systems, Applied Mathematical Sciences, Vol. 64 (Springer-Verlag, New York, 1987) p. 354.

${ }^{65} \mathrm{G}$. Froyland, "Computer-assisted bounds for the rate of decay of correlations," Commun. Math. Phys. 189, 237-257 (1997).

${ }^{66}$ A. Pikovsky and O. Popovych, "Persistent patterns in deterministic mixing flows," Europhys. Lett. 61, 625-631 (2003).

${ }^{67} \mathrm{P}$. Koltai, "A stochastic approach for computing the domain of attraction without trajectory simulation," in Dynamical Systems, Differential Equations and Applications, 8th AIMS Conference. Suppl., Vol. 2 (2011) pp. 854-863.

${ }^{68}$ V. Rossi, E. Ser-Giacomi, C. Lopez, and E. Hernandez-Garcia, "Hydrodynamic provinces and oceanic connectivity from a transport network help designing marine reserves," Geophys. Res. Let. 41, 2883-2891 (2014).

${ }^{69}$ E. Ser-Giacomi, V. Rossi, C. Lopez, and E. Hernandez-Garcia, "Flow networks: A characterization of geophysical fluid transport," Chaos 25, 036404 (2015).

${ }^{70}$ M.-H. Rio, S. Guinehut, and L. Gilles, "New CNES-CLS09 global mean dynamic topography computed from the combination of GRACE data, altimetry, and in situ measurements," Journal of Geophysical Research 116, C07018 (2011).

${ }^{71}$ P.-Y. Le Traon, D. Antoine, A. Bentamy, H. Bonekamp, L. Breivik, B. Chapron, G. Corlett, G. Dibarboure, P. DiGiacomo, C. Donlon, Y. Faugere, J. Font, F. Girard-Ardhuin, F. Gohin, J. Johannessen, M. Kamachi, G. Lagerloef, J. Lambin, G. Larnicol, P. L. Borgne, E. Leuliette, E. Lindstrom, M. Martin, E. Maturi, L. Miller, L. Mingsen, R. Morrow, N. Reul, M. Rio, H. Roquet, R. Santoleri, and J. Wilkin, "Use of satellite observations for operational oceanography: recent achievements and future prospects," Journal of Operational Oceanography 8, s12-s27 (2015).

${ }^{72}$ L. L. Fu, D. B. Chelton, P.-Y. Le Traon, and R. Morrow, "Eddy dynamics from satellite altimetry," Oceanography 23, 14-25 (2010).

${ }^{73}$ F. Vivier and C. Provost, "Direct velocity measurements in the Malvinas Current," Journal of Geophysical Research: Oceans 104, 21083-21103 (1999), https://agupubs.onlinelibrary.wiley.com/doi/pdf/10.1029/1999JC900163.

${ }^{74}$ F. J. Beron-Vera, M. J. Olascoaga, and G. J. Goni, "Oceanic mesoscale vortices as revealed by Lagrangian coherent structures," Geophys. Res. Lett. 35, L12603 (2008). 
${ }^{75}$ A. J. H. M. Reniers, J. H. MacMahan, F. J. Beron-Vera, and M. J. Olascoaga, "Ripcurrent pulses tied to Lagrangian coherent structures," Geophys. Res. Lett. 37, L05605 (2010).

${ }^{76}$ R. P. Mied, G. J. Lindemann, and J. C. McWilliams, "The generation and evolution of mushroom-like vortices," Journal of Physical Oceanography 21, 489-510 (1991), https://doi.org/10.1175/1520-0485(1991)021;0489:TGAEOM ¿2.0.CO;2.

${ }^{77}$ D. Etling, D. Hansen, and R. Jurrens, "The development of mushroom-like vortices from shear flow instabilities," Dynamics of Atmospheres and Oceans 20, 107-126 (1993).

${ }^{78}$ J. A. Smith and J. L. Largier, "Observations of nearshore circulation: Rip currents," J. Geophys. Res. 100, 10967-10975 (1995).

${ }^{79}$ A. Pascual, Y. Faugere, G. Larnicol, and P.-Y. Le Traon, "Improved description of the ocean mesoscale variability by combining four satellite altimeters," Geophys. Res. Lett. 33, L02611 (2006).

${ }^{80}$ F. J. Beron-Vera, M. J. Olascoaga, and G. J. Goni, "Surface ocean mixing inferred from different multisatellite altimetry measurements," J. Phys. Oceanogr. 40, 2466-2480 (2010).

${ }^{81}$ D. Orúe-Echevarría, J. L. Pelegrí, F. Machín, A. Hernández-Guerra, and M. Emelianov, "Inverse modeling the Brazil-Malvinas Confluence," Journal of Geophysical Research: Oceans 124, 527-554 (2019).

${ }^{82}$ R. M. Samelson, "Fluid exchange across a meandering jet," J. Phys. Oceanogr. 22, 431440 (1992).

${ }^{83}$ V. I. Arnold, V. V. Kozlov, and A. I. Neishtadt, "Mathematical aspects of classical and celestial mechanics," in Dynamical Systems III, Encyclopedia of Mathematical Sciencies, Vol. 3 (Springer-Verlag, Berlin Heidelberg, 2006) 3rd ed., p. 518.

${ }^{84}$ R. Lumpkin and M. Pazos, "Measuring surface currents with Surface Velocity Program drifters: the instrument, its data and some recent results," in Lagrangian Analysis and Prediction of Coastal and Ocean Dynamics, edited by A. Griffa, A. D. Kirwan, A. Mariano, T. Özgökmen, and T. Rossby (Cambridge University Press, 2007) Chap. 2, pp. 39-67.

${ }^{85}$ A. L. Sybrandy and P. P. Niiler, "WOCE/TOGA Lagrangian drifter contruction manual," Tech. Rep. SIO Reference 91/6 (Scripps Institution of Oceanography, La Jolla, California, 1991). 
${ }^{86}$ R. Lumpkin, S. A. Grodsky, L. Centurioni, M.-H. Rio, J. A. Carton, and D. Lee, "Removing spurious low-frequency variability in drifter velocities," J. Atm. Oce. Tech. 30, 353-360 (2012).

${ }^{87}$ F. J. Beron-Vera, M. J. Olascoaga, and R. Lumpkin, "Inertia-induced accumulation of flotsam in the subtropical gyres," Geophys. Res. Lett. 43, 12228-12233 (2016).

${ }^{88} \mathrm{G}$. Froyland, "An analytic framework for identifying finite-time coherent sets in timedependent dynamical systems," Physica D 250, 1-19 (2013).

${ }^{89}$ J. H. LaCasce, "Statistics from Lagrangian observations," Progr. Oceanogr. 77, 1-29 (2008).

${ }^{90}$ A. N. Maximenko, J. Hafner, and P. Niiler, "Pathways of marine debris derived from trajectories of Lagrangian drifters," Mar. Pollut. Bull. 65, 51-62 (2012).

${ }^{91}$ R. Tarjan, "Depth-first search and linear graph algorithms," SIAM J. Comput. 1, 146-160 (1972).

${ }^{92}$ G. Froyland, C. P. Rock, and K. Sakellariou, "Sparse eigenbasis approximation: Multiple feature extraction across spatiotemporal scales with application to coherent set identification," Commun Nonlinear Sci Numer Simulat 77, 81-107 (2019).

${ }^{93} \mathrm{Z}$. Hu, Y. W. G. Pan, and Z. Wu, "Sparse principal component analysis via rotation and truncation," IEEE Trans. Neural Networks Learn. Syst. 27, 875-890 (2016).

${ }^{94}$ P. Brémaud, Markov chains, Gibbs Fields Monte Carlo Simulation Queues, Texts in Applied Mathematics, Vol. 31 (Springer, New York, 1999).

${ }^{95}$ M. Dellnitz, G. Froyland, C. Horenkam, K. Padberg-Gehle, and A. Sen Gupta, "Seasonal variability of the subpolar gyres in the southern ocean: a numerical investigation based on transfer operators," Nonlinear Process. Geophys. 16, 655-663 (2009).

${ }^{96}$ M. Saraceno, C. Provost, and A. R. Piola, "On the relationship of satellite retrieved surface temperature fronts and chlorophyll- $a$ in the Western South Atlantic," J. Geophys. Res. 110, C11016 (2005).

${ }^{97}$ M. Saraceno, C. Provost, and M. Lebbah, "Biophysical Regions identification using an artificial neuronal network: a case study in the South Western Atlantic," Advances in Space Research 37, 793-805 (2006).

${ }^{98}$ H. R. Cho, T. G. Shepherd, and V. A. Vladimirov, "Application of the direct Liapunov method to the problem of symmetric stability in the atmosphere," Journal of the Atmospheric Sciences 50, 822-836 (1993). 
${ }^{99} \mathrm{M} . \mathrm{Mu}, \mathrm{T}$. G. Shepherd, and K. Swanson, "On nonlinear symmetric stability and the nonlinear saturation of symmetric instability," J. Atmos. Sci. 53, 2918-2923 (1996).

${ }^{100}$ B. J. Hoskins, "The role of potential vorticty in symmetric stability and instability," Quart. J. R. Met. Soc. 100, 480-482 (1974).

${ }^{101}$ P. M. Saunders and B. A. King, "Bottom currents derived from a shipborne ADCP on WOCE cruise A11 in the South Atlantic," J. Phys. Oceanogr. 25, 329-347 (1995).

${ }^{102}$ H. Aref, "Stirring by chaotic advection," J. Fluid Mech. 143, 1-21 (1984).

${ }^{103}$ D. Valla and A. R. Piola, "Evidence of upwelling events at the northern Patagonian shelf break," Journal of Geophysical Research: Oceans 120, 7635-7656 (2015).

${ }^{104}$ M. M. Carranza, S. T. Gille, A. R. Piola, M. Charo, and S. I. Romero, "Wind modulation of upwelling at the shelf-break front off Patagonia: Observational evidence," Journal of Geophysical Research: Oceans 122, 2401-2421 (2017).

${ }^{105}$ T. Zhang, A. E. Yankovsky, A. R. Piola, and D. Valla, "Observations of semidiurnal internal tides on the patagonian shelf," Continental Shelf Research 167, 46-54 (2018).

${ }^{106}$ I. Hernández-Carrasco, A. Orfila, V. Rossi, and V. Garçon, "Effect of small scale transport processes on phytoplankton distribution in coastal seas," Scientific Reports 8, 8613 (2018). ${ }^{107}$ J. M. Magalhães and J. C. B. da Silva, "Internal waves along the Malvinas Current: Evidence of transcritical generation in satellite imagery," Oceanography 30, 110-119 (2017). ${ }^{108}$ L. N. Thomas, J. R. Taylor, E. A. D’Asaro, C. M. Lee, J. M. Klymak, and A. Shcherbina, "Symmetric instability, inertial oscillations, and turbulence at the gulf stream front," Journal of Physical Oceanography 46, 197-217 (2016). 


$$
\begin{aligned}
& \text { red yellow blue } \\
& \rho 0.97630 .7797 \quad 0.9835 \\
& \tau 7.79521 .151811 .4850
\end{aligned}
$$

Table I. Retention probability $(\rho)$ and time ( $\tau$, in months) inside each of the three provinces of the Lagrangian geography in Fig. 6, which are labelled by their colors in the figure. 

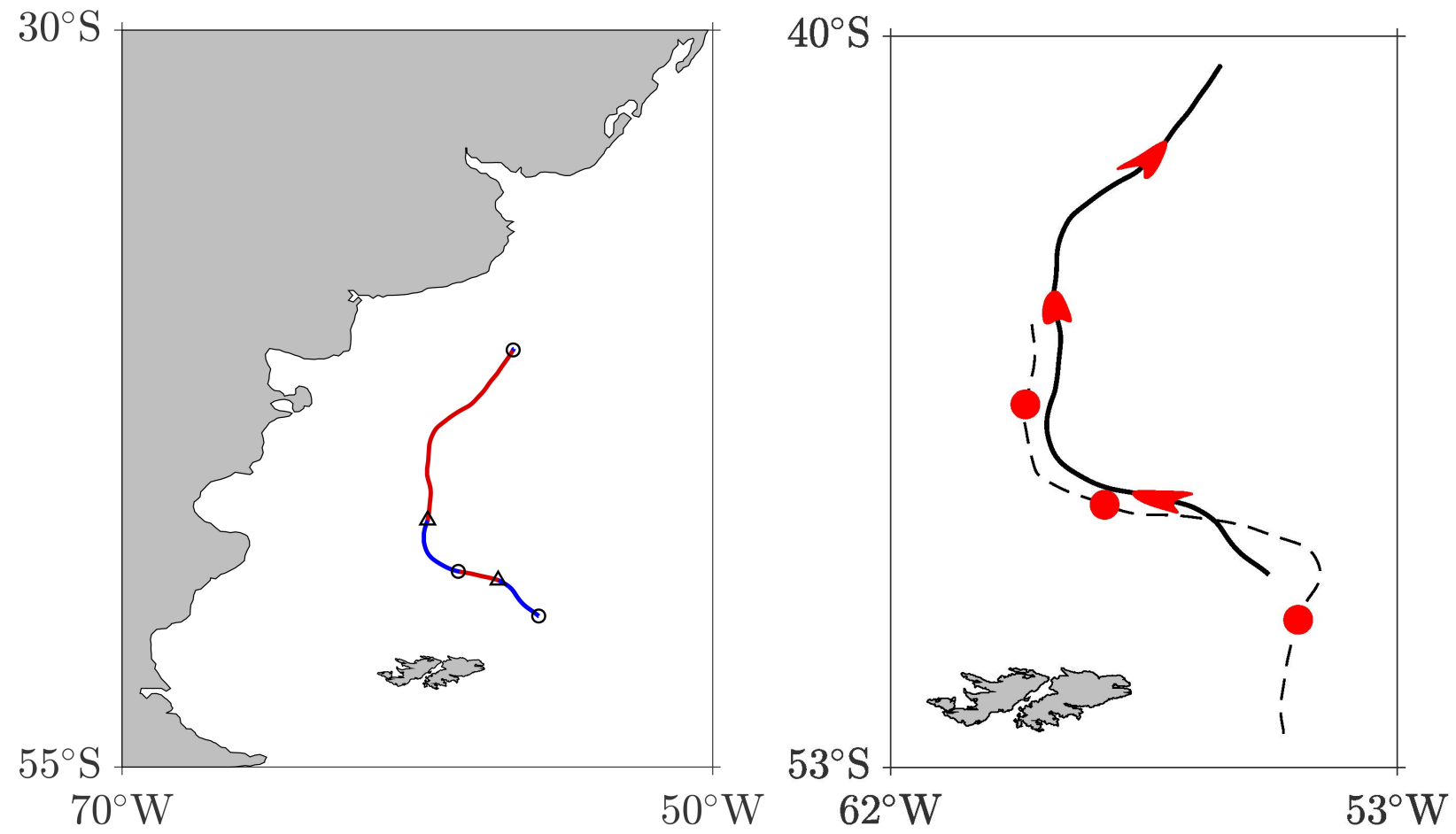

Figure 1. (left) Shearless-parabolic LCS extracted from altimetry-derived velocity on $t_{0}=$ 12 Dec 2001 using backward integration with $T=-15$ days. Portions of the LCS colored red and blue are nearly neutral squeezing and stretching tensorline segments of the Cauchy-Green tensor field, respectively, connecting wedge and trisector singularities of the field, indicated [by triangles and circles, respectively. (right) Overlaid on the LCS (solid), forward-advected images at $t_{0}$ (V shapes) of circles axially straddling the backward-advected image of the LCS at $t_{0}-|T|$ (dashed). 


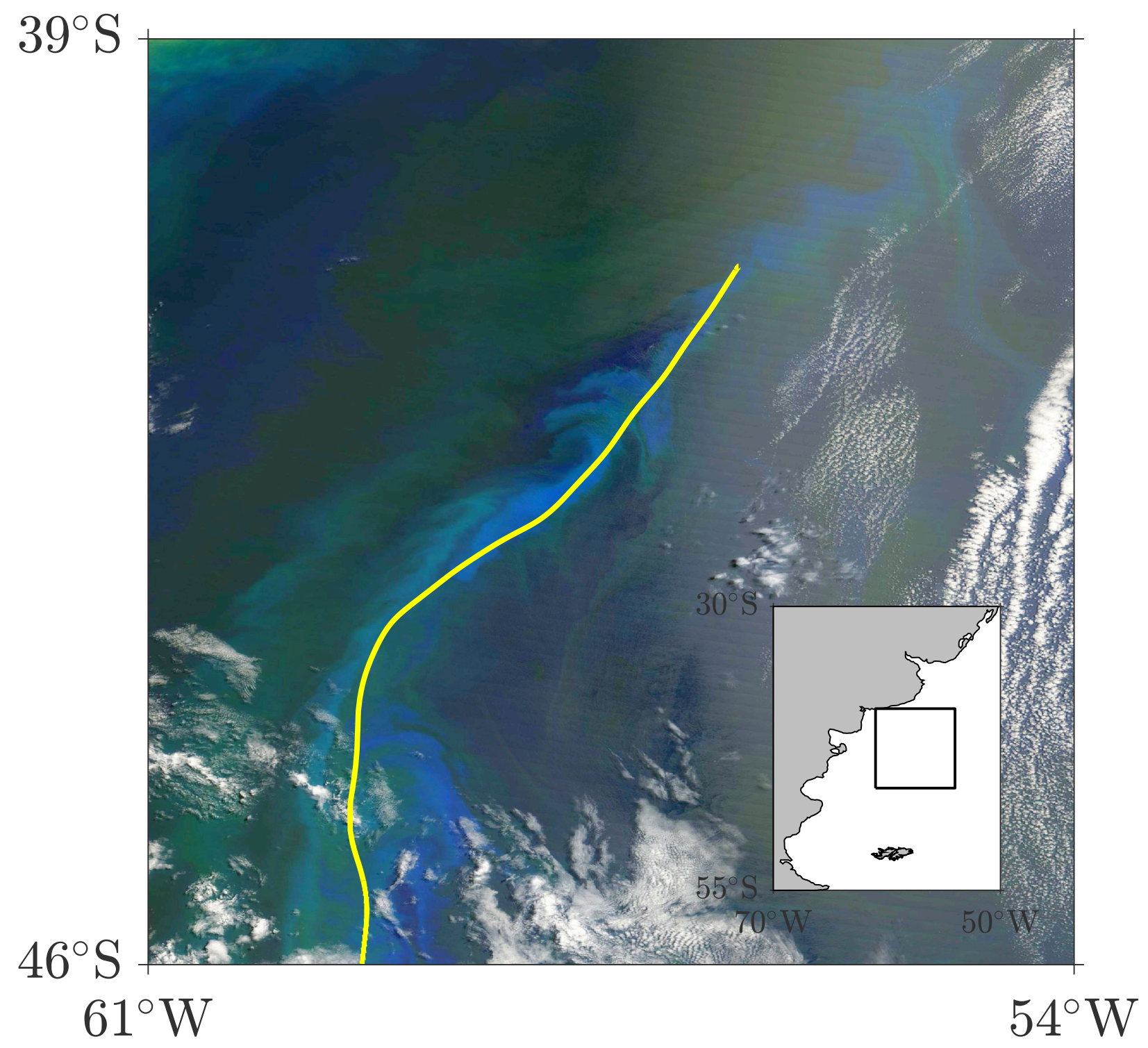

Figure 2. Ocean pseudo color image on 12 Dec 2001 derived from the Moderate Resolution Imaging Spectroradiometer (MODIS) aboard Terra with the shearless-parabolic LCS extracted from altimetry overlaid. (Image obtained from NASA Goddard Space Flight Center, Ocean Ecology Laboratory, Ocean Biology Processing Group; (2014): Sea-viewing Wide Field-of-view Sensor (SeaWiFS) Ocean Color Data, NASA OB.DAAC, doi:10.5067/ORBVIEW-2/allowbreak SEAWIFS_OC.2014.0. Accessed on 29 Sep 2018.) 


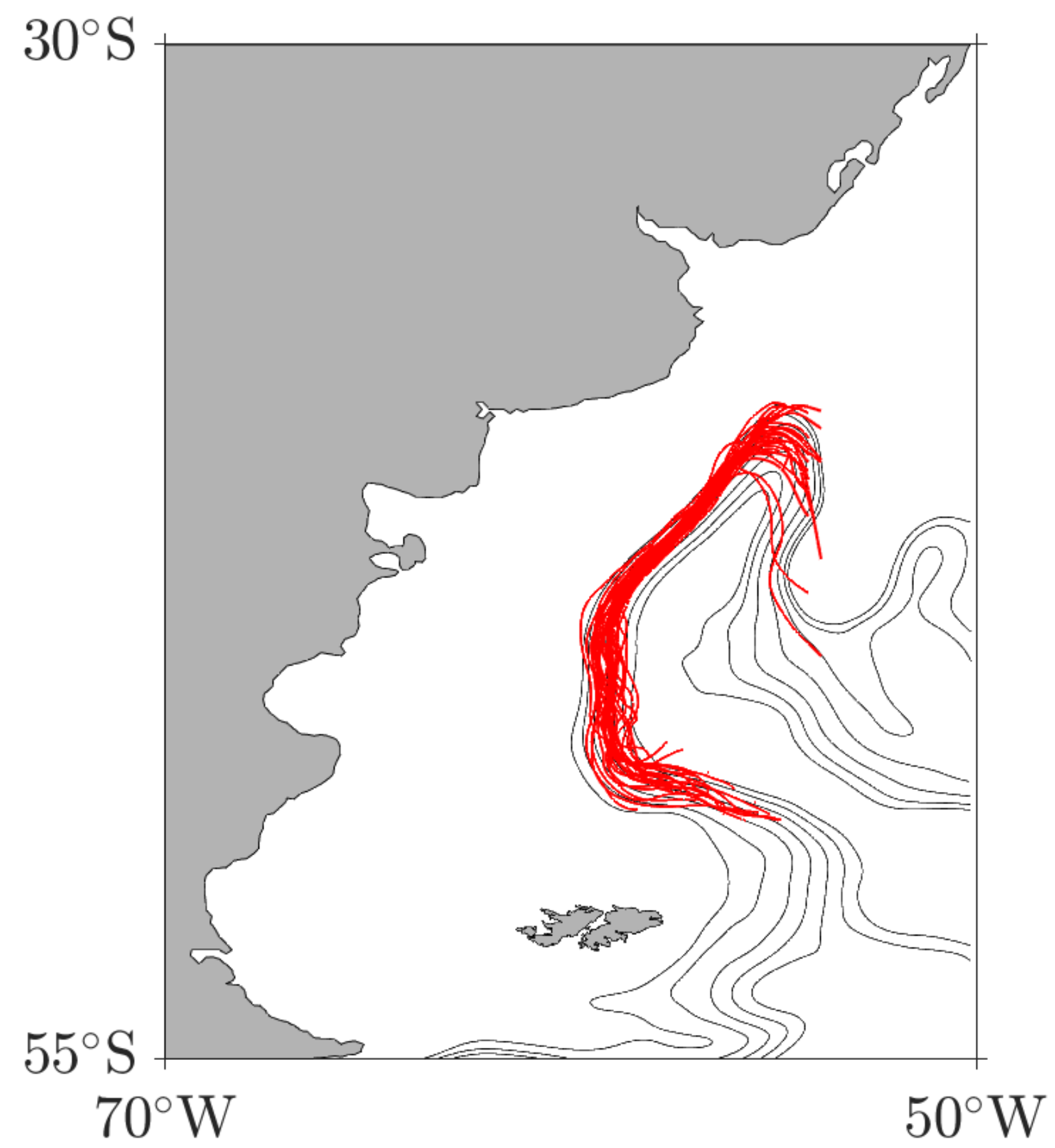

Figure 3. Mean (15 Oct 2002-15 Sep 2005) streamlines along the Malvinas current core (black) overlaid with shearless-parabolic LCS extracted over windows $\left[t_{0}+T, t_{0}\right]$ with $t_{0}$ sliding monthly over 30 Oct $2002-15$ Sep 2005 and $T=-15$ days. 

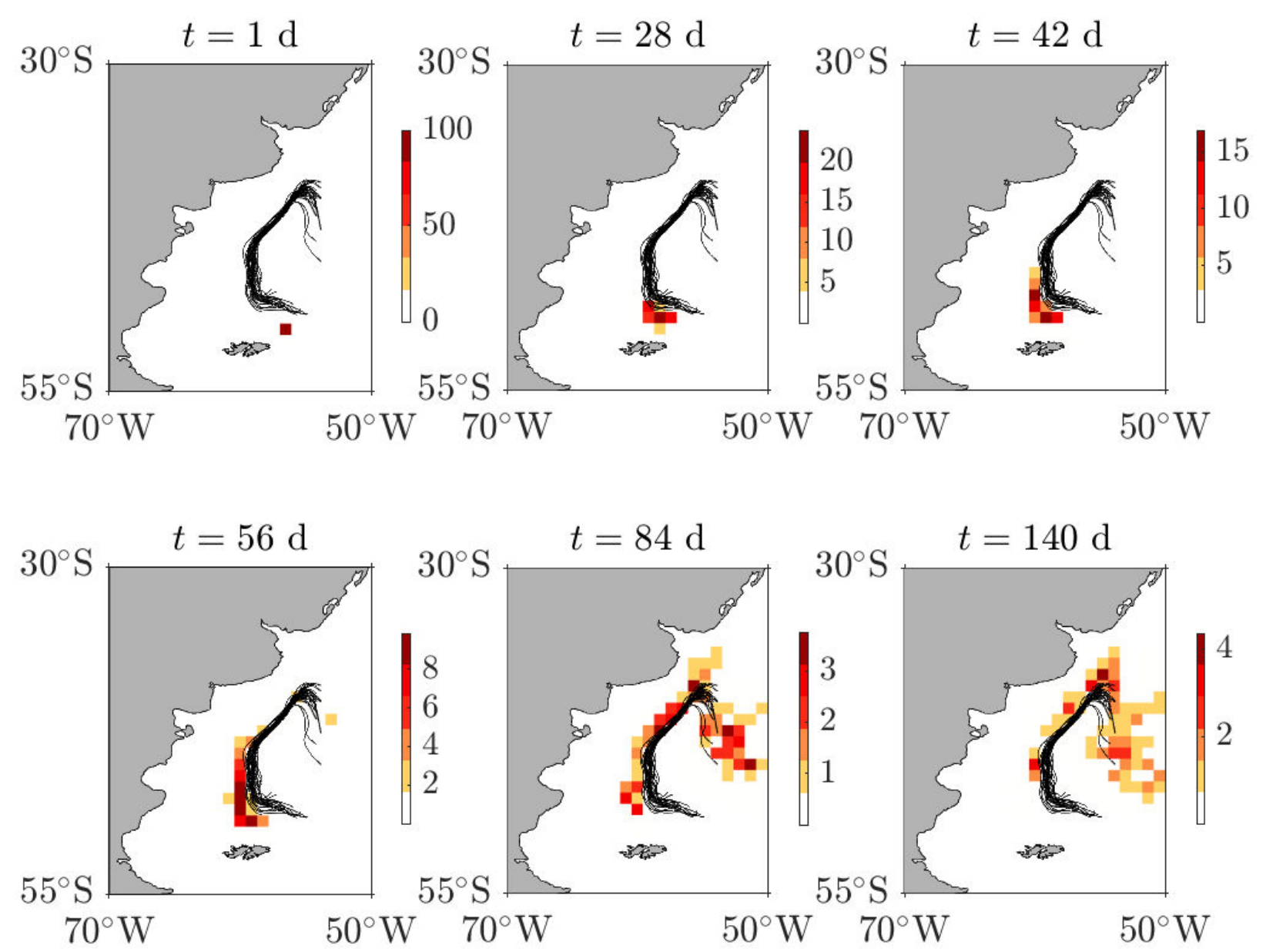

Figure 4. Snapshots of the ensemble-mean evolution over 15 Oct 2002-15 Sep 2005 of tracers under the altimetry-derived flow with the extracted shearless-parabolic LCS overlaid. The percentage of tracer particles visiting the boxes of a grid covering the domain is shown. 

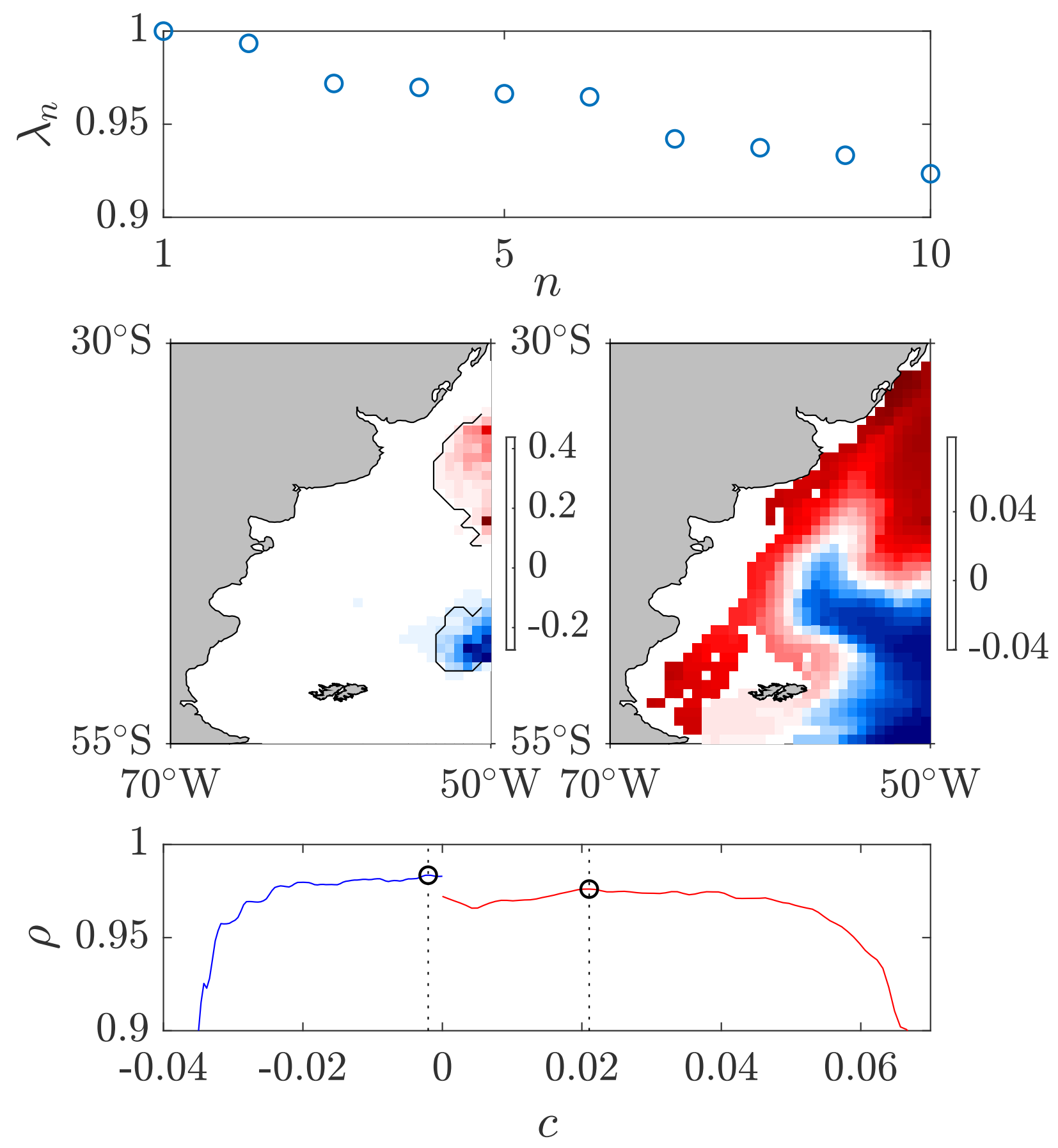

Figure 5. (top) A portion of the discrete eigenspectrum of the drifter-based transition matrix $P$ showing the top 15 real eigenvalues. (middle) Left and right $\left(\mathbf{r}_{2}\right)$ eigenvectors of $P$ with largest none unity eigenvalue $\left(\lambda_{2}=0.9946\right)$. (bottom) Probability of a trajectory to be retained within regions where $\mathbf{r}_{2}<c$ if $c<0$ or $\mathbf{r}_{2}>c$ if $c>0$ conditioned on starting in those regions. 


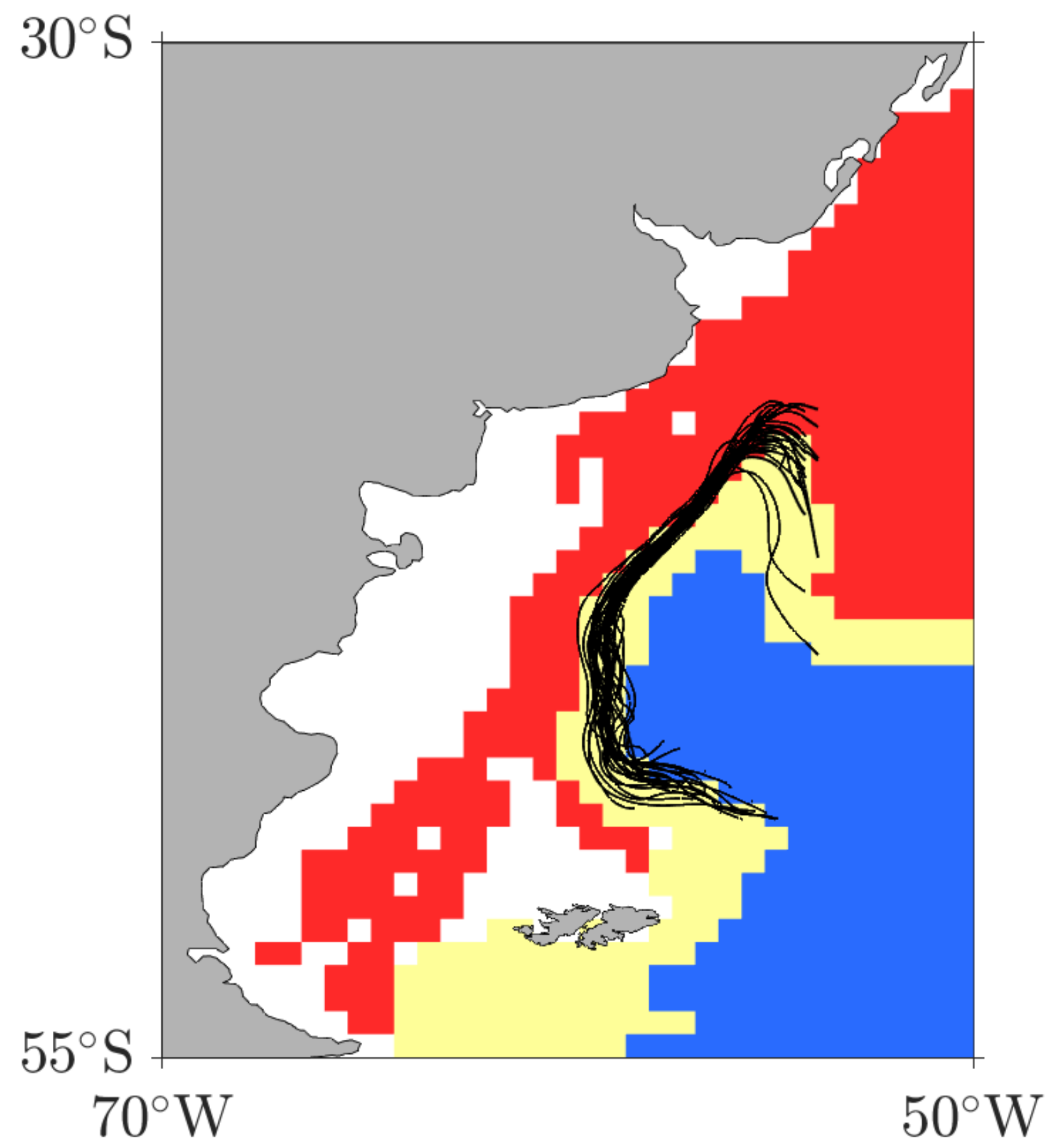

Figure 6. Lagrangian geography deduced from the structure of the dominant right eigenvector of the drifter-based transition matrix $P$ overlaid with all shearless-parabolic LCS extracted from satellite altimetry. Retention probability and time are maximized in the red and blue provinces, which represent the main Lagrangian provinces, while the yellow province represents a transition region with smaller retention probability and time (cf. Table I). 

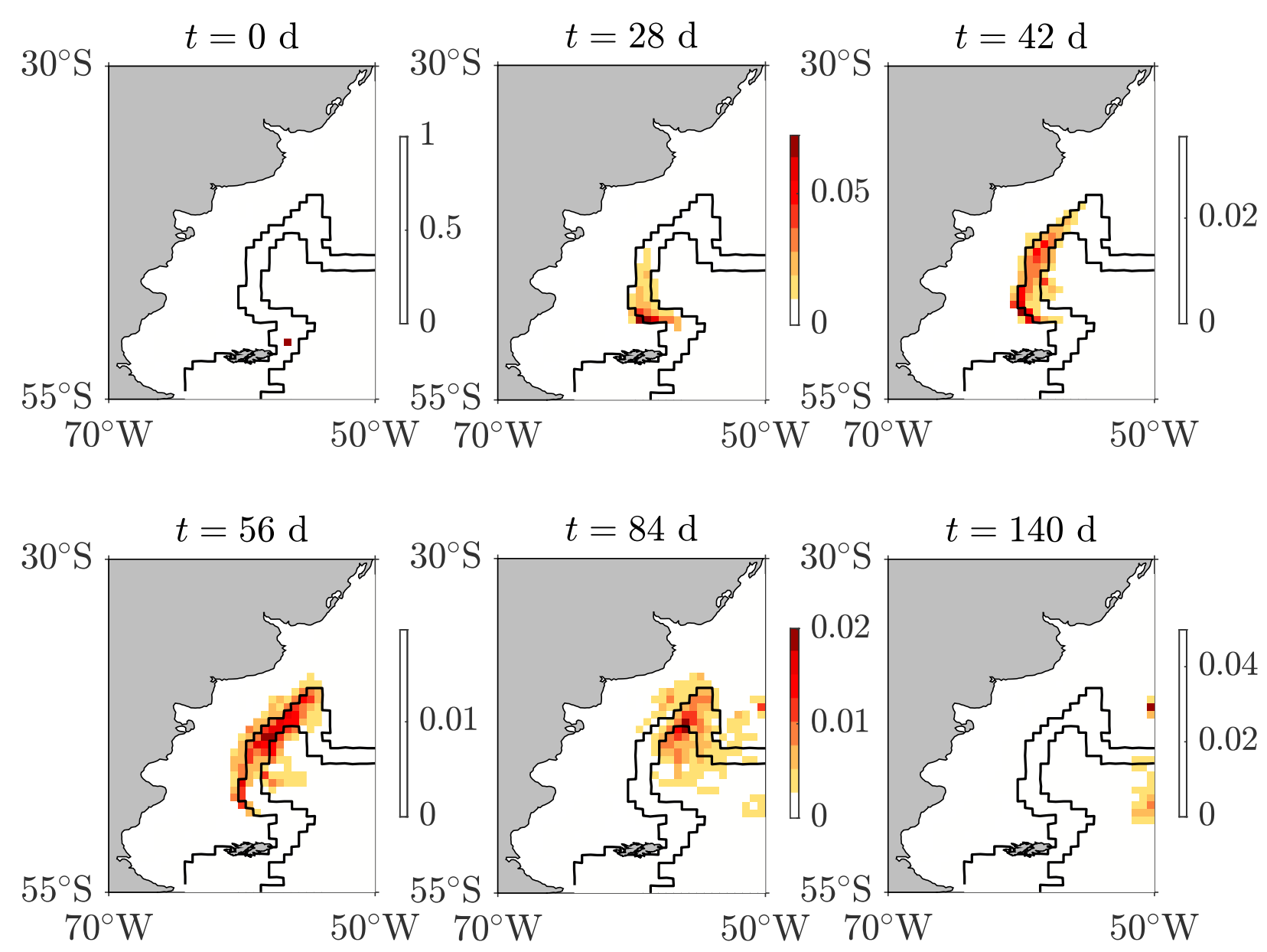

Figure 7. Snapshots of the drifter-based forward evolution of a probability density with the boundaries of the main Lagrangian provinces indicated. 

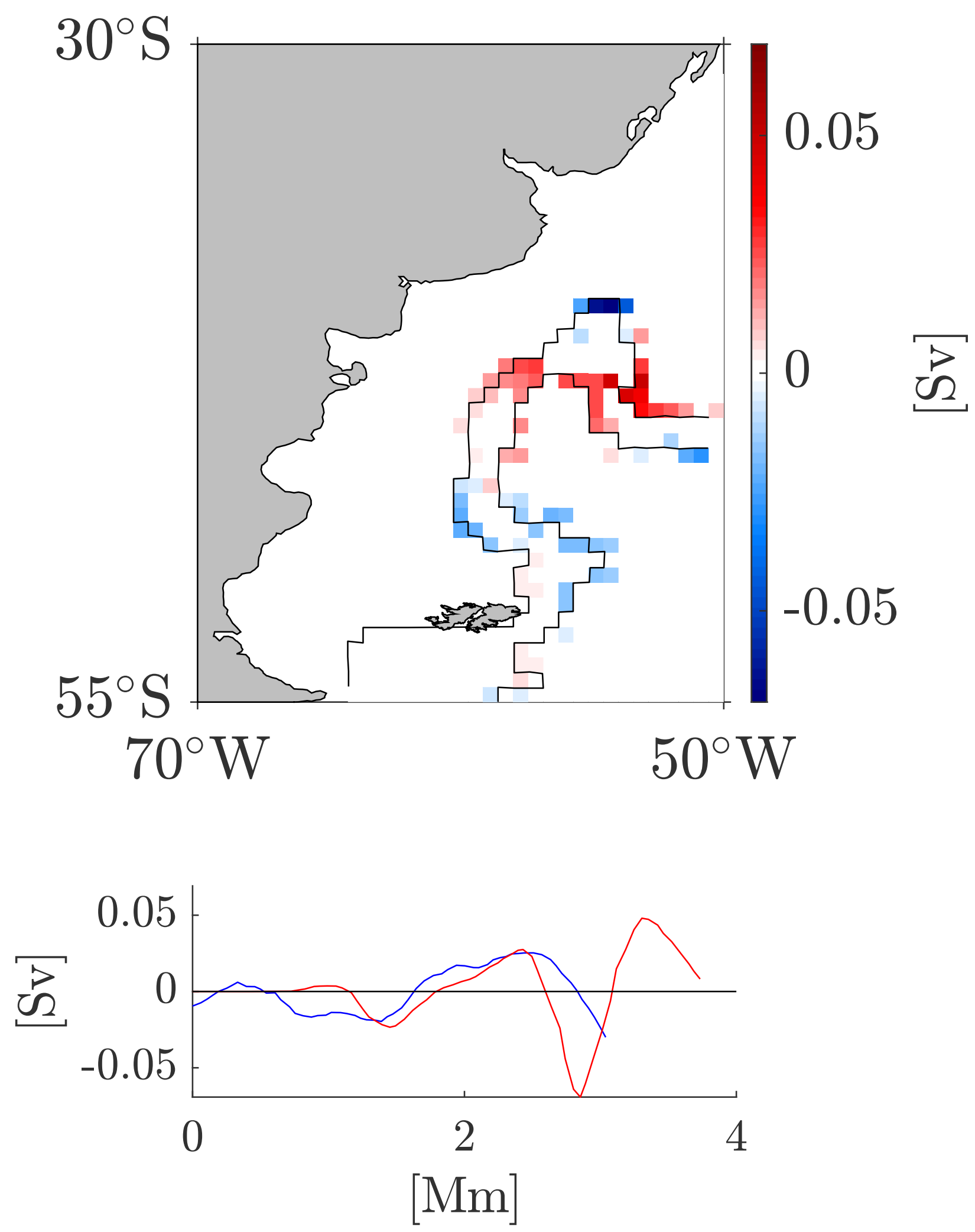

Figure 8. (top) Drifter-based estimate of the flux across the boundaries of the transition province of the Lagrangian geography. (bottom) The flux shown as a function of arclength (increasing northward) along each boundary. The red (blue) curve corresponds to the boundary with the main province painted red (blue) in Fig. 6. 

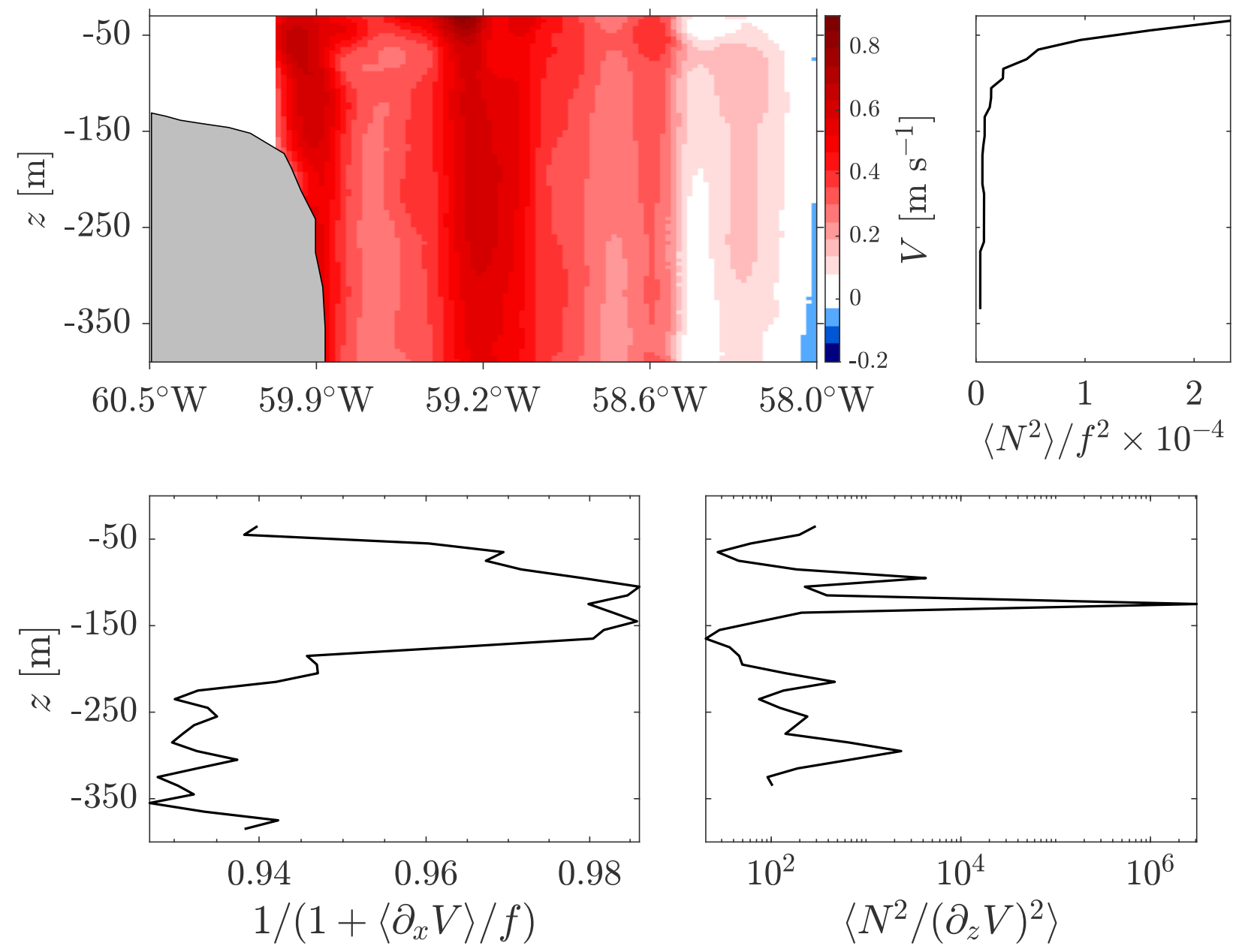

Figure 9. (top left) Meridional velocity section along $45^{\circ} \mathrm{S}$ across the Malvinas Current from a hull mounted ADCP collected on 28 Dec 1992 from RSS Discovery during WOCE cruise A11. (top right) Normalized by the Coriolis parameter squared, along-transect average of Brunt-Väis̈ala frequency squared computed using temperature and salinity from CTD casts during WOCE A11. (bottom left) Along-transect-average of the ratio of the Coriolis parameter and the vertical component of the absolute vorticity. (bottom right) Along-transect-average of Richardson number. 


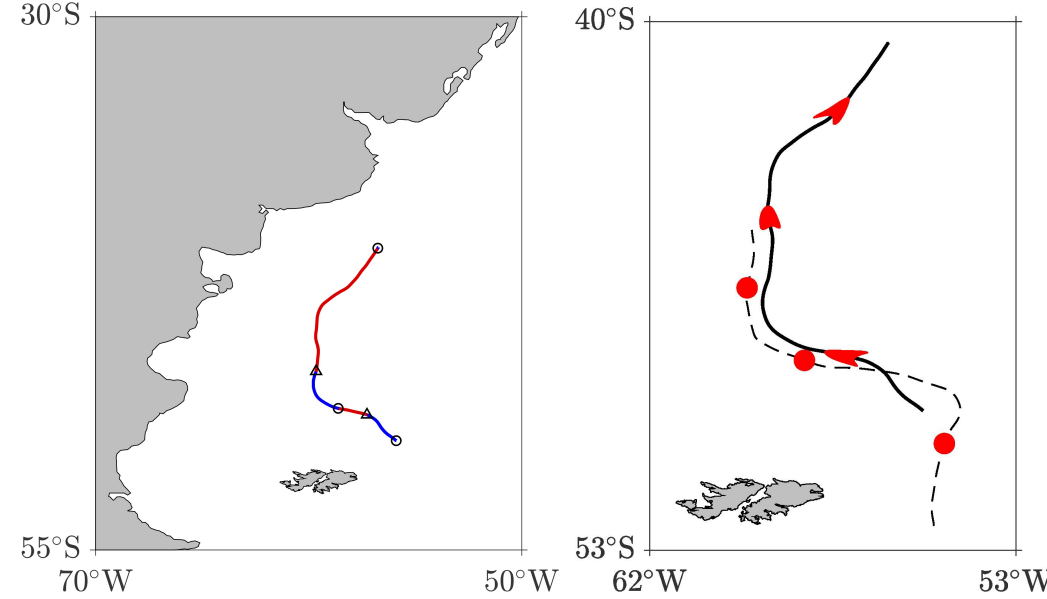

崖

這

으 ్ำ

变

응 응

이응

으

등 흥

닝

要

픙

步

힐

우 빵

풍

荡

崖

崖

$\frac{1}{0}$ 
d) $\frac{\omega}{1}$

우탄

蒙它

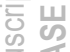

를 땀

U
0
U

\%
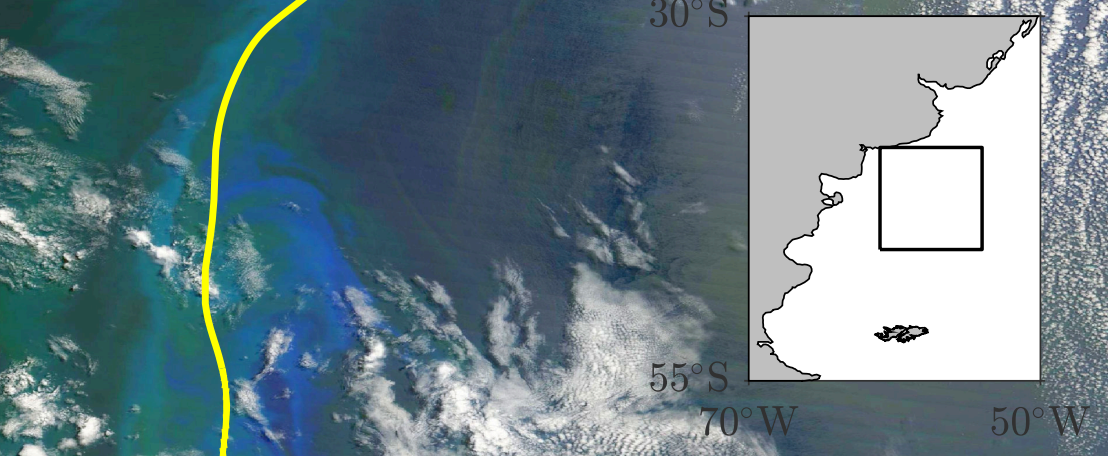
$30^{\circ} \mathrm{S}$

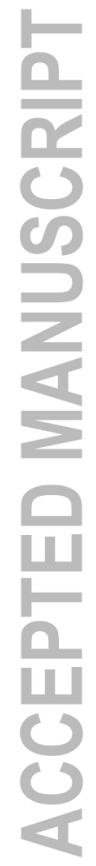

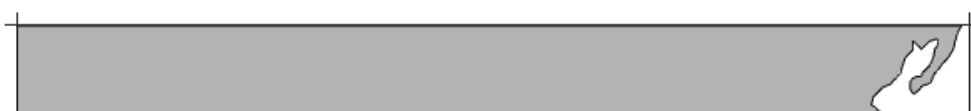

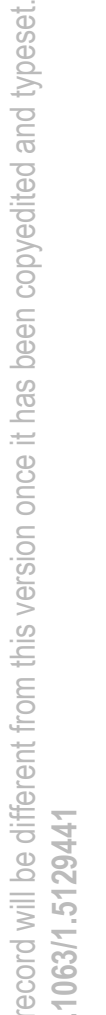

$55^{\circ} \mathrm{S}$

$70^{\circ} \mathrm{W}$

$50^{\circ} \mathrm{W}$

世

늠

$\cong \frac{1}{4}$

히

우 땀

논

穵

들

름

0

잉 


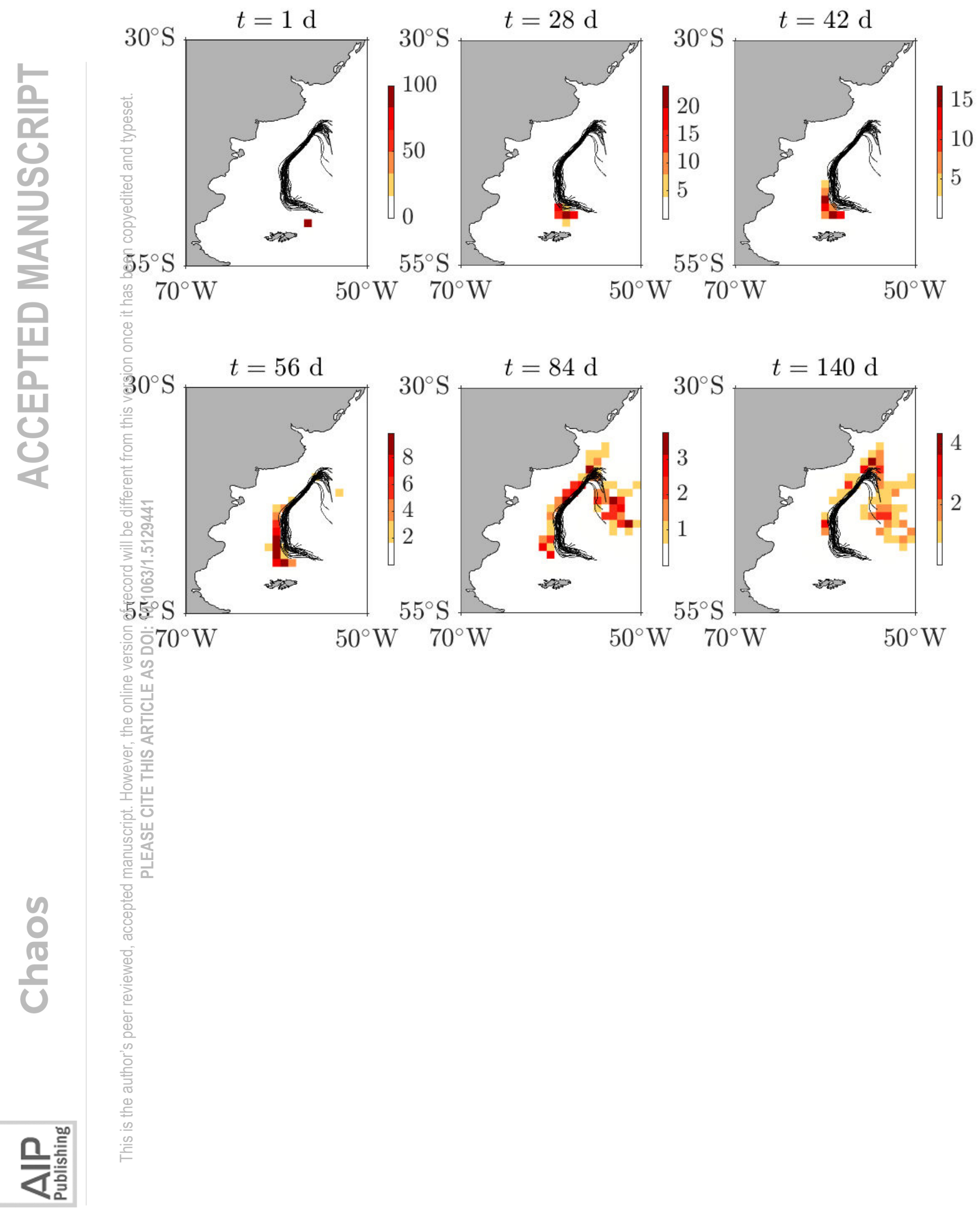


$30^{\circ} \mathrm{S}$

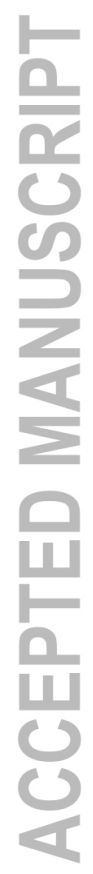

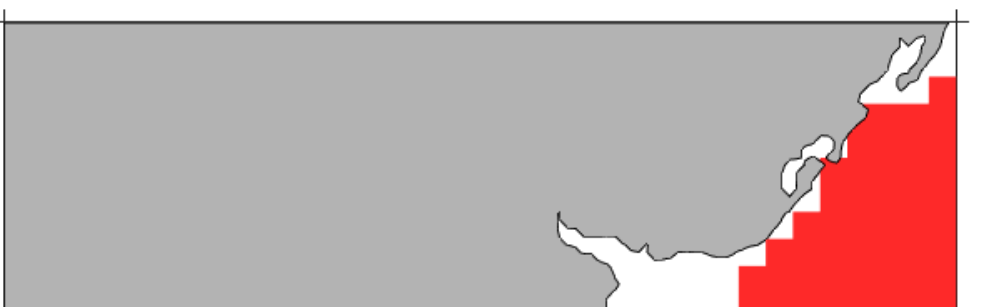

$5{ }^{\circ} \mathrm{S}$

哭觉 $70^{\circ} \mathrm{W}$

\%

픙

음

$\cong \frac{1}{4}$

히 을

우트

吉

는

들

है

ปั

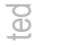




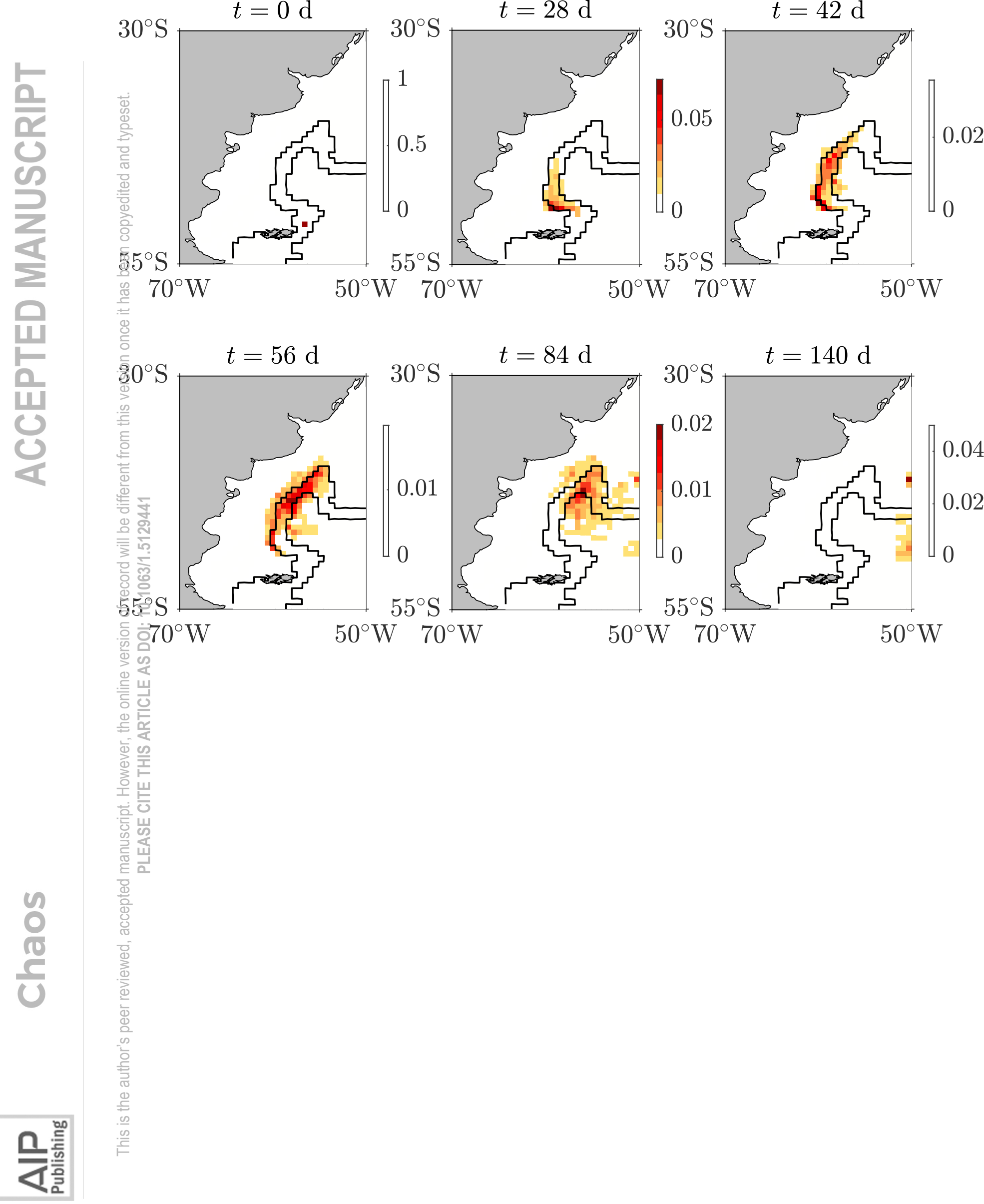



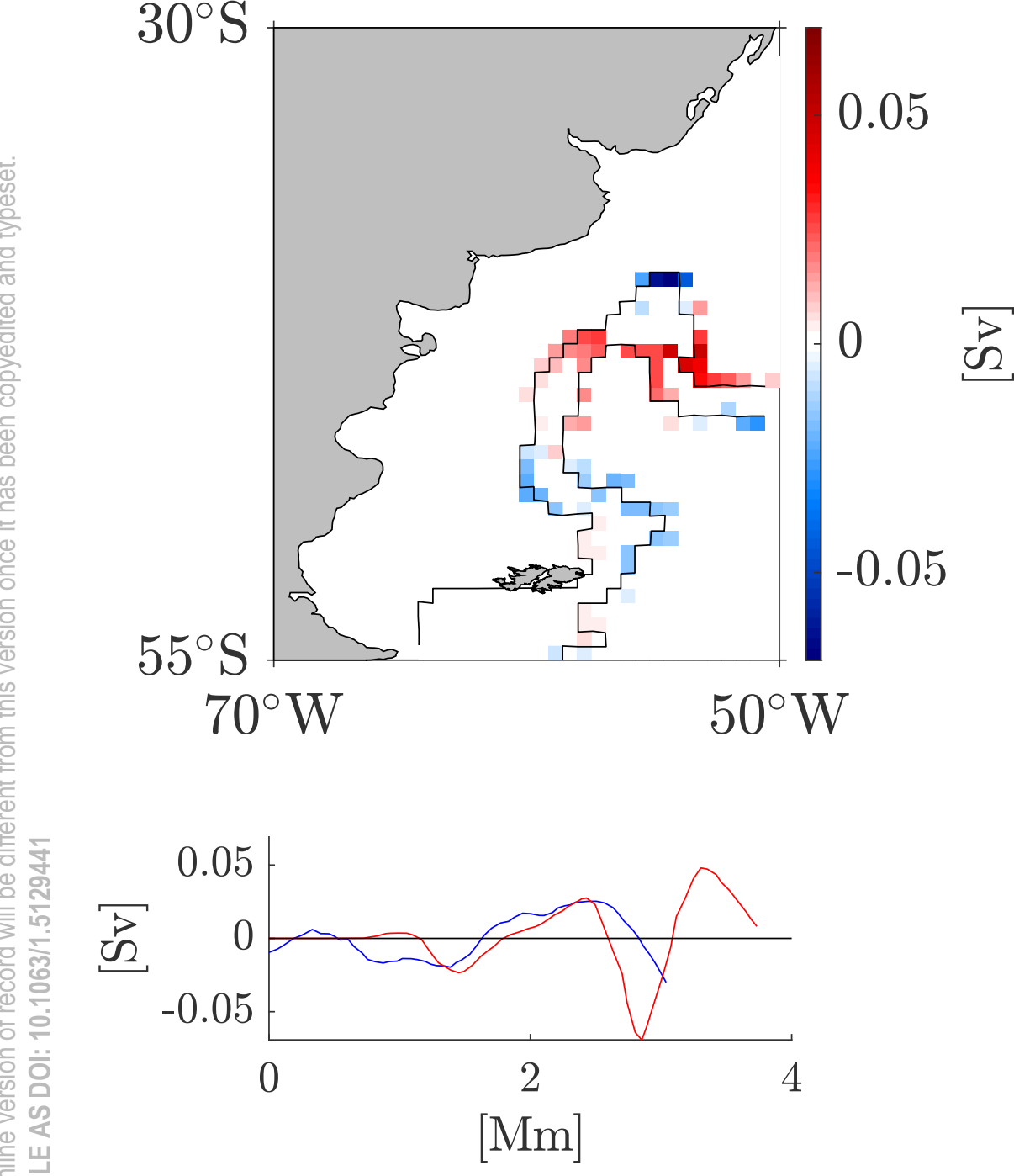


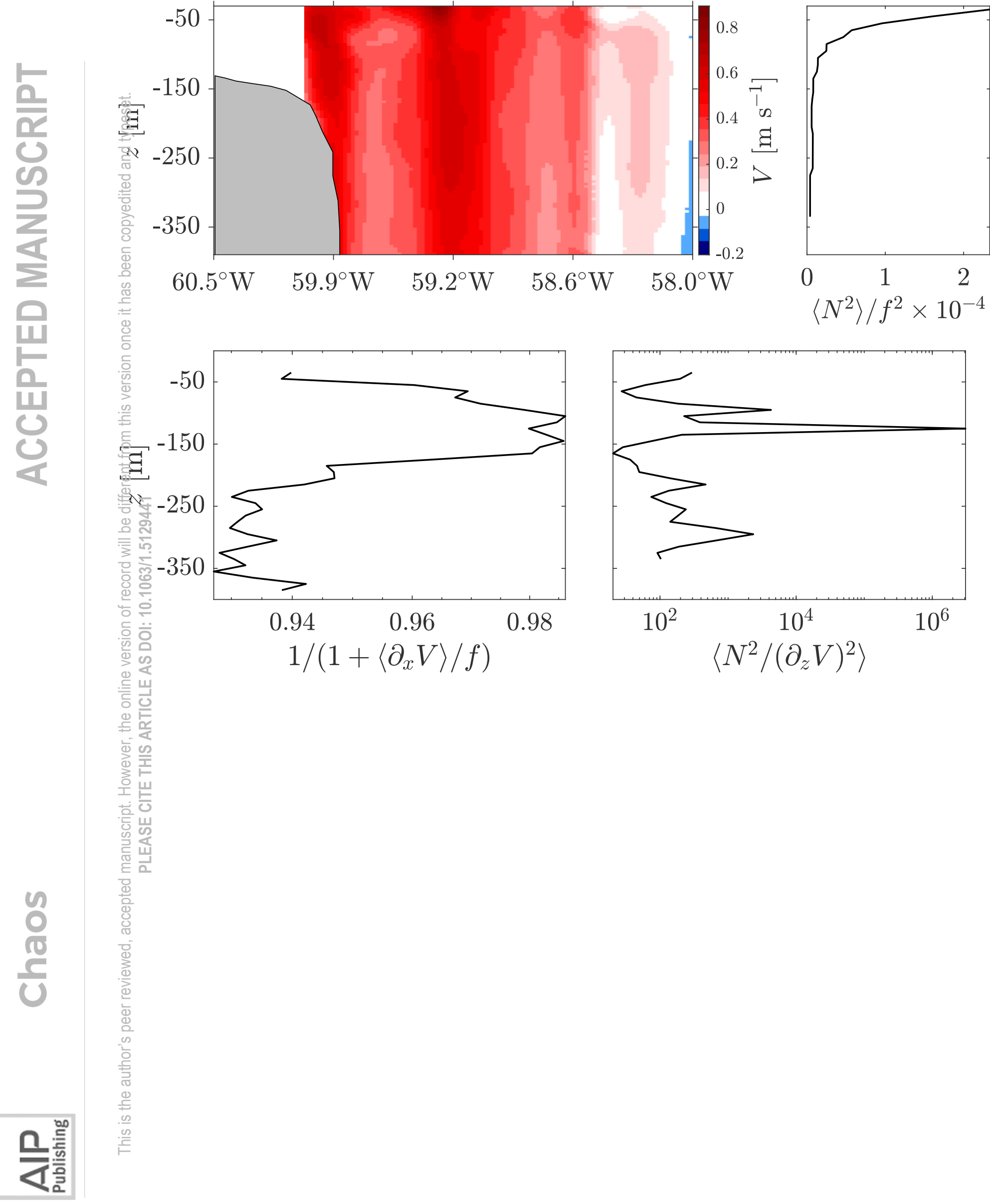

